\title{
Charge Radii and Quadrupole Moments of the Low-Lying Baryons in the Chiral Constituent Quark Model
}

\author{
Neetika Sharma and Harleen Dahiya \\ Department of Physics, Dr. B.R. Ambedkar National Institute of Technology, Jalandhar 144011, India \\ Correspondence should be addressed to Harleen Dahiya; dahiyah@nitj.ac.in
}

Received 16 December 2012; Revised 15 April 2013; Accepted 13 May 2013

Academic Editor: Jan E. Alam

Copyright ( 2013 N. Sharma and H. Dahiya. This is an open access article distributed under the Creative Commons Attribution License, which permits unrestricted use, distribution, and reproduction in any medium, provided the original work is properly cited.

The chiral constituent quark model ( $\chi \mathrm{CQM})$ with general parameterization method (GPM) has been formulated to calculate the charge radii of the spin $(1 / 2)^{+}$octet and $(3 / 2)^{+}$decuplet baryons and quadrupole moments of the spin $(3 / 2)^{+}$decuplet baryons and spin $(3 / 2)^{+} \rightarrow(1 / 2)^{+}$transitions. The implications of such a model have been investigated in detail for the effects of symmetry breaking and GPM parameters pertaining to the one-, two-, and three-quark contributions. Our results are not only comparable with the latest experimental studies but also agree with other phenomenological models. It is found that the $\chi \mathrm{CQM}$ is successful in giving a quantitative and qualitative description of the charge radii and quadrupole moments.

\section{Introduction}

The internal structure of baryons is determined in terms of electromagnetic Dirac and Pauli form factors $F_{1}\left(Q^{2}\right)$ and $F_{2}\left(Q^{2}\right)$ or equivalently in terms of the electric and magnetic Sachs form factors $G_{E}\left(Q^{2}\right)$ and $G_{M}\left(Q^{2}\right)[1]$. The electromagnetic form factors are the fundamental quantities of theoretical and experimental interest which are further related to the static low energy observables of charge radii and magnetic moments. One of the main challenges in the theoretical and experimental hadronic physics is to understand the structure of hadrons within the quantum chromodynamics (QCD) in terms of these moments. Although QCD is accepted as the fundamental theory of strong interactions, the direct prediction of these kinds of observables from the first principle of QCD still remains a theoretical challenge as they lie in the nonperturbative regime of QCD.

Following the discoveries that the quarks and antiquarks carry only $30 \%$ of the total proton spin $[2,3]$, the orbital angular momentum of quarks and gluons is expected to make a significant contribution. In addition to this, there is a significant contribution coming from the strange quarks in the nucleon which are otherwise not present in the valence structure. It therefore becomes interesting to discuss the interplay between the spin of nonvalence quark and the orbital angular momentum in understanding the spin structure of baryons. Further, the experimental developments [4-6], providing information on the radial variation of the charge, and magnetization densities of the proton give the evidence for the deviation of the charge distribution from spherical symmetry. On the other hand, it is well known that the quadrupole moment of the nucleon should vanish on account of its spin-1/2 nature. This observation has naturally turned to be the subject of intense theoretical and experimental activity.

The mean square charge radius $\left(r_{\mathrm{B}}^{2}\right)$, giving the possible "size" of baryon, has been investigated experimentally with the advent of new facilities at JLAB, SELEX Collaborations [7-13]. Several measurements have been made for the charge radii of $p, n$, and $\Sigma^{-}$in electron-baryon scattering experiments $[13,14]$ giving $r_{p}=0.877 \pm 0.007 \mathrm{fm}\left(r_{p}^{2}=0.779 \pm\right.$ $\left.0.025 \mathrm{fm}^{2}[15]\right)$ and $r_{n}^{2}=-0.1161 \pm 0.0022 \mathrm{fm}^{2}[12]$. The recent measurement of $r_{\Sigma^{-}}^{2}[13,14]$ is particularly interesting as it gives the first estimate for the charge form factor of a strange baryon at low momentum transfer.

The $\Delta(1232)$ resonance is the lowest-lying excited state of the nucleon in which the search for quadrupole strength 
has been carried out [16-19]. The spin and parity selection rules in the $\gamma+p \rightarrow \Delta^{+}$transition allow three contributing photon absorption amplitudes, the magnetic dipole $G_{M 1}$, the electric quadrupole moment $G_{E 2}$, and the charge quadrupole moment $G_{C 2}$. The $G_{M 1}$ amplitude gives us information on magnetic moment, whereas the information on the intrinsic quadrupole moment can be obtained from the measurements of $G_{E 2}$ and $G_{C 2}$ amplitudes [12]. If the charge distribution of the initial and final three-quark states was spherically symmetric, the $G_{E 2}$ and $G_{C 2}$ amplitudes of the multipole expansion would be zero [20]. However, the recent experiments at Mainz, Bates, Bonn, and JLab Collaborations [7$11,21,22]$ reveal that these quadrupole amplitudes are clearly nonzero [12]. The ratio of electric quadrupole amplitude to the magnetic dipole amplitude is at least $E 2 / M 1 \equiv-0.025 \pm$ 0.005 , and a comparable value of same sign and magnitude has been measured for the C2/M1 ratio [12]. Further, the quadrupole transition moment $\left(Q^{\Delta^{+} N}\right)$ measured by LEGS and Mainz collaborations $\left(-0.108 \pm 0.009 \pm 0.034 \mathrm{fm}^{2}[16-\right.$ 18] and $-0.0846 \pm 0.0033 \mathrm{fm}^{2}$ [19], resp.) also leads to the conclusion that the nucleon and the $\Delta^{+}$are intrinsically deformed.

The naive quark model (NQM) [23-27] is one of the simplest model to describe the hadron properties and interactions in the low energy regime. This model is able to provide a simple intuitive picture of the hadron structure in terms of three valence quarks (qqq) for baryons and quark antiquark $(q \bar{q})$ for mesons. It allows the direct calculations of the low energy hadronic matrix elements including their spectra and successfully accounts for many of the low energy properties of the hadrons in terms of the valence quarks [28-32]. Interestingly, with the inclusion of the spin-spin interactions generated configuration mixing [28] between the valence quarks, the NQM has not only given an accurate description of the hadron spectroscopy data but also has been able to describe some subtle features of the data including the $N-\Delta$ mass difference, photohelicity amplitudes, and baryon magnetic moments [29-36]. However, some major findings on the experimental front discussed below have brought into prominence the inadequacies of the NQM.

The measurements in the deep inelastic scattering (DIS) experiments $[2,3]$ indicate that the valence quarks of the proton carry only about $30 \%$ of its spin and also establishe the asymmetry of the quark distribution functions [37-41]. This is referred to as the "proton spin problem" in NQM. Several effective and phenomenological models have been developed to explain the "proton spin problem" by including spontaneous breaking of chiral symmetry and have been further applied to study the electromagnetic properties of baryons.

For the calculations pertaining to the baryon charge radii, NQM leads to vanishing charge radii for the neutral baryons like $n, \Sigma^{0}, \Xi^{0}$, and $\Lambda$. This is in contradiction to the experimental data. The inclusion of quark spin-spin interactions in NQM modify the baryon wavefunction to some extent leading to the breaking of the $\mathrm{SU}(3)$ symmetry and a nonvanishing neutron charge mean square radius [29-32]. A likely cause of these dynamical shortcomings is that the NQM does not respect chiral symmetry whose spontaneous breaking leads to the emission of Goldstone bosons (GBs). In this context, it becomes important to incorporate the effect of chiral symmetry breaking $(\chi \mathrm{SB})$ in the phenomenological models to obtain a reasonable agreement with the data. On the other hand, a wide variety of accurately measured data have been accumulated for the static low energy properties of baryons, for example, masses, electromagnetic moments, charge radii, and low energy dynamical properties such as scattering lengths and decay rates, which has renewed considerable interest in the low energy baryon spectroscopy. The direct calculations of these quantities from the first principle of QCD are extremely difficult as they require the nonperturbative methods. Several effective and phenomenological models such as Lattice QCD, effective field theories, QCD sum rules, and variants of quark models have been developed to explain the failures of the NQM and further applied to study the properties of baryons.

Some of the important models measuring the charge radii of octet baryon are the Skyrme model with bound state approach [42-44], slow-rotor approach [45], semibosonized SU(3) NJL model [46], cloudy bag model [47], variants of constituent quark models [48-54], $1 / N_{c}$ expansion approach [55-58], perturbative chiral quark model $(\mathrm{P} \chi \mathrm{QM})$ [59], heavy-baryon chiral perturbation theory $(\mathrm{HB} \chi \mathrm{PT})$ [60], chiral perturbation theory $(\chi \mathrm{PT})[61,62]$, Lattice QCD [63], and so forth. The charge radii of decuplet baryons have been studied within the framework of Lattice QCD [64-66], quark model [67], $1 / N_{c}$ expansion $[55,56]$, chiral perturbation theory [68], and so forth. The results for different theoretical models are however not consistent with each other.

There have been a lot of theoretical investigations in understanding the implications of the C2/M1 and E2/M1 ratios in finding out the exact sign of deformation in the $\operatorname{spin}(1 / 2)^{+}$octet baryons. However, there is a little consensus between the results even with respect to the sign of the nucleon deformation. Some of the models predict the deformation in nucleon as oblate $[69,70]$, some predict a prolate nucleon deformation [71-80] whereas others speak about "deformation" without specifying the sign. The quadrupole moment of the $(3 / 2)^{+}$decuplet baryons has also been studied using the variants of the constituent quark model (CQM) [81-84], chiral quark soliton model ( $\chi \mathrm{QSM})$ [85], spectator quark model [86-88], slow-rotator approach (SRA) [89, 90], skyrme model [91, 92], general parametrization method [93, 94], light cone QCD sum rules (QCDSR) [95, 96], large $N_{c}[97,98]$, chiral perturbation theory $(\chi \mathrm{PT})$ [99103], Lattice QCD (LQCD) [104-108], and so forth. In this case also, the results for different theoretical models are not consistent in terms of sign and magnitude with each other.

As the hadron structure is sensitive to the pion cloud in the low energy regime, a coherent understanding is necessary as it will provide a test for the QCD-inspired effective field theories. One of the important nonperturbative approaches which finds its application in the low energy regime is the chiral constituent quark model $(\chi \mathrm{CQM})[109$, 
110]. It is one of the most convenient languages for the treatment of light hadrons at low energies using the effective interaction Lagrangian approach of the strong interactions. The $\chi$ CQM coupled with the "quark sea" generation through the chiral fluctuation of a constituent quark GBs [111119] successfully explains the "proton spin problem" [117119], hyperon $\beta$ decay parameters $[120,121]$, strangeness content in the nucleon [122-124], magnetic moments of octet and decuplet baryons including their transitions [125-127], magnetic moments of $(1 / 2)^{-}$octet baryon resonances [128], magnetic moments of $(1 / 2)^{-}$and $(3 / 2)^{-} \Lambda$ resonances [129], charge radii [130], quadrupole moment [131], and so forth. The model is successfully extended to predict the important role played by the small intrinsic charm (IC) content in the nucleon spin in the SU(4) $\chi \mathrm{CQM}[132]$ and to the calculate the magnetic moment of spin $(1 / 2)^{+}$and spin $(3 / 2)^{+}$charm baryons including their radiative decays [133]. In view of the above developments in the $\chi \mathrm{CQM}$, it become desirable to extend the model to calculate the charge radii and quadrupole moment of the spin $(1 / 2)^{+}$octet and spin $(3 / 2)^{+}$decuplet baryons.

The purpose of the present communication is to calculate the charge radii of the spin $(1 / 2)^{+}$octet and spin $(3 / 2)^{+}$decuplet baryons and quadrupole moment of the spin $(3 / 2)^{+}$decuplet baryons including the spin $(3 / 2)^{+} \rightarrow$ $(1 / 2)^{+}$transitions within the framework of $\chi$ CQM using a general parametrization method (GPM) [134-138]. In order to understand the role of pseudoscalar mesons in the baryon charge radii and quadrupole moment, we will compare our results with NQM as well as other phenomenological models. The detailed analysis of SU(3) symmetry breaking would also be carried out in the $\chi \mathrm{CQM}$. Further, we aim to discuss the implications of GPM parameters by calculating the extent to which the three-quark term contributes.

\section{Charge Radii and Quadrupole Moments}

The mean square charge radii $\left(r_{\mathrm{B}}^{2}\right)$ and quadrupole moments $\left(Q_{B}\right)$ are the lowest order moments of the charge density $\rho$ in a low-momentum expansion. The charge radii contain fundamental information about the possible "size" of the baryons whereas the "shape" of a spatially extended particle is determined by its quadrupole moment [139-142].

The mean square charge radius $r_{\mathrm{B}}^{2}$ of a given baryon is a scalar under spatial rotation and is defined as

$$
\left\langle r^{2}\right\rangle=\int d^{3} r \rho(\mathbf{r}) r^{2}
$$

where $\rho(\mathbf{r})$ is the charge density. The intrinsic quadrupole moment with respect to the body frame of axis is defined as

$$
Q_{0}=\int d^{3} \mathbf{r} \rho(\mathbf{r})\left(3 z^{2}-r^{2}\right)
$$

For the charge density concentrated along the $z$-direction, the term proportional to $3 z^{2}$ dominates, $Q_{0}$ is positive, and the particle is prolate shaped. If the charge density is concentrated in the equatorial plane perpendicular to $z$ axis, the term proportional to $r^{2}$ dominates, $Q_{0}$ is negative, and the particle is oblate shaped.

The most general form of the multipole expansion of the nucleon charge density $\rho$ in the spin-flavor space can be expressed as

$$
\begin{gathered}
\rho=\mathrm{A}^{\prime} \sum_{i=1}^{3} e_{i} \mathbf{1}-\mathrm{B}^{\prime} \sum_{i \neq j}^{3} e_{i}\left[2 \boldsymbol{\sigma}_{i} \cdot \boldsymbol{\sigma}_{j}-\left(3 \boldsymbol{\sigma}_{i z} \boldsymbol{\sigma}_{j z}-\boldsymbol{\sigma}_{i} \cdot \boldsymbol{\sigma}_{j}\right)\right] \\
-\mathrm{C}^{\prime} \sum_{i \neq j \neq k}^{3} e_{i}\left[2 \boldsymbol{\sigma}_{j} \cdot \boldsymbol{\sigma}_{k}-\left(3 \boldsymbol{\sigma}_{j z} \boldsymbol{\sigma}_{k z}-\boldsymbol{\sigma}_{j} \cdot \boldsymbol{\sigma}_{k}\right)\right] .
\end{gathered}
$$

The charge radii operator composed of the sum of one-, two-, and three-quark terms is expressed as

$$
\widehat{r^{2}}=\mathrm{A} \sum_{i=1}^{3} e_{i} \mathbf{1}+\mathrm{B} \sum_{i \neq j}^{3} e_{i} \boldsymbol{\sigma}_{i} \cdot \boldsymbol{\sigma}_{j}+\mathrm{C} \sum_{i \neq j \neq k}^{3} e_{i} \boldsymbol{\sigma}_{j} \cdot \boldsymbol{\sigma}_{k},
$$

whereas the quadrupole moment operator composed of a two- and three-quark term can be expressed as

$$
\begin{aligned}
\widehat{\mathrm{Q}}= & \mathrm{B}^{\prime} \sum_{i \neq j}^{3} e_{i}\left(3 \sigma_{i z} \sigma_{j z}-\boldsymbol{\sigma}_{i} \cdot \boldsymbol{\sigma}_{j}\right) \\
& +\mathrm{C}^{\prime} \sum_{i \neq j \neq k}^{3} e_{i}\left(3 \sigma_{j z} \sigma_{k z}-\boldsymbol{\sigma}_{j} \cdot \boldsymbol{\sigma}_{k}\right) .
\end{aligned}
$$

The coefficients called GPM parameters of the charge radii and quadrupole moments are related to each other as $\mathrm{A}=$ $A^{\prime}, B=-2 B^{\prime}$, and $C=-2 C^{\prime}$. These GPM parameters are to be determined from the experimental observations on charge radii and quadrupole moment.

Before calculating the matrix elements corresponding to the charge radii and quadrupole moment, it is essential to simplify various operator terms involved in (4). It can be easily shown that

$$
\begin{gathered}
\sum_{i \neq j} e_{i}\left(\boldsymbol{\sigma}_{\mathbf{i}} \cdot \boldsymbol{\sigma}_{\mathbf{j}}\right)=2 \mathbf{J} \cdot \sum_{i} e_{i} \boldsymbol{\sigma}_{\mathbf{i}}-3 \sum_{i} e_{i}, \\
\sum_{i \neq j \neq k} e_{i}\left(\boldsymbol{\sigma}_{\mathbf{j}} \cdot \boldsymbol{\sigma}_{\mathbf{k}}\right)= \pm 3 \sum_{i} e_{i}-\sum_{i \neq j} e_{i}\left(\boldsymbol{\sigma}_{\mathbf{i}} \cdot \boldsymbol{\sigma}_{\mathbf{j}}\right),
\end{gathered}
$$

where +ve sign holds for $J=3 / 2$ and - ve sign for $J=1 / 2$ states leading to different operators for spin $(1 / 2)^{+}$and spin $(3 / 2)^{+}$baryons:

\begin{tabular}{c|c|c} 
& & \\
\hline Operator & $\sum_{i \neq j} e_{i}\left(\boldsymbol{\sigma}_{\mathbf{i}} \cdot \boldsymbol{\sigma}_{\mathbf{j}}\right)$ & $\sum_{i \neq j \neq k} e_{i}\left(\boldsymbol{\sigma}_{\mathbf{j}} \cdot \boldsymbol{\sigma}_{\mathbf{k}}\right)$ \\
\hline$J=\frac{1}{2}$ & $3 \sum_{i} e_{i} \boldsymbol{\sigma}_{\mathbf{i z}}-3 \sum_{i} e_{i}$ & $-3 \sum_{i} e_{i} \boldsymbol{\sigma}_{\mathbf{i z}}$ \\
$J=\frac{3}{2}$ & $5 \sum_{i} e_{i} \boldsymbol{\sigma}_{\mathbf{i z}}-3 \sum_{i} e_{i}$ & $6 \sum_{i} e_{i}-5 \sum_{i} e_{i} \boldsymbol{\sigma}_{\mathbf{i z}}$ \\
\hline & &
\end{tabular}


The charge radii operators for the spin $(1 / 2)^{+}$octet and spin $(3 / 2)^{+}$decuplet baryons can now be expressed as

$$
\begin{gathered}
\widehat{r_{\mathrm{B}}^{2}}=(\mathrm{A}-3 \mathrm{~B}) \sum_{i} e_{i}+3(\mathrm{~B}-\mathrm{C}) \sum_{i} e_{i} \sigma_{i z} \\
\widehat{r_{\mathrm{B}^{*}}^{2}}=(\mathrm{A}-3 \mathrm{~B}+6 \mathrm{C}) \sum_{i} e_{i}+5(\mathrm{~B}-\mathrm{C}) \sum_{i} e_{i} \sigma_{\mathrm{iz}}
\end{gathered}
$$

It is clear from the above equations that the determination of charge radii basically reduces to the evaluation of the flavor $\left(\sum_{i} e_{i}\right)$ and spin $\left(\sum_{i} e_{i} \sigma_{i z}\right)$ structure of a given baryon. The charge radii squared $r_{\mathrm{B}\left(\mathrm{B}^{*}\right)}^{2}$ for the octet (decuplet) baryons can now be calculated by evaluating matrix elements corresponding to the operators in (8) and (9) and are given as

$$
r_{\mathrm{B}}^{2}=\left\langle\mathrm{B}\left|\widehat{r_{\mathrm{B}}^{2}}\right| \mathrm{B}\right\rangle, \quad r_{\mathrm{B}^{*}}^{2}=\left\langle\mathrm{B}^{*}\left|\widehat{\mathrm{r}^{*}}{ }^{2}\right| \mathrm{B}^{*}\right\rangle .
$$

Here $|\mathrm{B}\rangle$ and $\left|\mathrm{B}^{*}\right\rangle$, respectively, denote the spin-flavor wavefunctions for the spin $(1 / 2)^{+}$octet and the spin $(3 / 2)^{+}$ decuplet baryons.

The quadrupole moment operators for the spin $(1 / 2)^{+}$, $\operatorname{spin}(3 / 2)^{+}$baryons, and spin $(3 / 2)^{+} \rightarrow(1 / 2)^{+}$transitions can be calculated from the operator in (5) and are expressed as

$$
\begin{gathered}
\widehat{Q}_{\mathrm{B}}=\mathrm{B}^{\prime}\left(3 \sum_{i \neq j} e_{i} \sigma_{\mathrm{iz}} \sigma_{\mathrm{jz}}-3 \sum_{i} e_{i} \sigma_{\mathrm{iz}}+3 \sum_{i} e_{i}\right) \\
+\mathrm{C}^{\prime}\left(3 \sum_{i \neq j \neq k} e_{i} \sigma_{\mathrm{jz}} \sigma_{\mathrm{kz}}+3 \sum_{i} e_{i} \sigma_{\mathrm{iz}}\right), \\
\widehat{Q}_{\mathrm{B}^{*}}=\mathrm{B}^{\prime}\left(3 \sum_{i \neq j} e_{i} \sigma_{\mathrm{iz}} \sigma_{\mathrm{jz}}-5 \sum_{i} e_{i} \sigma_{\mathrm{iz}}+3 \sum_{i} e_{i}\right) \\
+\mathrm{C}^{\prime}\left(3 \sum_{i \neq j \neq k} e_{i} \boldsymbol{\sigma}_{\mathrm{jz}} \sigma_{\mathrm{kz}}+5 \sum_{i} e_{i} \sigma_{\mathrm{iz}}-6 \sum_{i} e_{i}\right), \\
\widehat{Q}_{\mathrm{B}^{*} \mathrm{~B}}=3 \mathrm{~B}^{\prime} \sum_{i \neq j} e_{i} \sigma_{\mathrm{iz}} \sigma_{\mathrm{jz}}+3 C^{\prime} \sum_{i \neq j \neq k} e_{i} \sigma_{\mathrm{jz}} \sigma_{\mathrm{kz}} \cdot
\end{gathered}
$$

It is clear from the above equations that the determination of quadrupole moment basically reduces to the evaluation of the flavor $\left(\sum_{i} e_{i}\right)$, spin $\left(\sum_{i} e_{i} \sigma_{\mathrm{iz}}\right)$, and tensor terms $\left(\sum_{i} e_{i} \boldsymbol{\sigma}_{\mathrm{iz}} \boldsymbol{\sigma}_{\mathrm{jz}}\right)$ and $\left(\sum_{i} e_{i} \sigma_{\mathbf{j z}} \sigma_{\mathbf{k z}}\right)$ for a given baryon.

Using the three-quark spin-flavor wavefunctions for the spin $(1 / 2)^{+}$octet and spin $(3 / 2)^{+}$decuplet baryons, the quadrupole moment can now be calculated by evaluating the matrix elements of operators in (11). We now have

$$
\begin{gathered}
Q_{B}=\left\langle B\left|\widehat{Q}_{B}\right| B\right\rangle, \quad Q_{B^{*}}=\left\langle B^{*}\left|\widehat{Q}_{B^{*}}\right| B^{*}\right\rangle, \\
Q_{B \rightarrow B^{*}}=\left\langle B^{*}\left|\widehat{Q}_{B^{*} B}\right| B\right\rangle .
\end{gathered}
$$

\section{Naive Quark Model (NQM)}

The appropriate operators for the spin and flavor structure of baryons in NQM are defined as

$$
\begin{aligned}
\sum_{i} e_{i} & =\sum_{q=u, d, s} n_{q}^{\mathrm{B}} q+\sum_{\bar{q}=\bar{u}, \bar{d}, \bar{s}} n_{\bar{q}}^{\mathrm{B}} \bar{q} \\
& =n_{u}^{\mathrm{B}} u+n_{d}^{\mathrm{B}} d+n_{s}^{\mathrm{B}} s+n_{\bar{u}}^{\mathrm{B}} \bar{u}+n_{\bar{d}}^{\mathrm{B}} \bar{d}+n_{\bar{s}}^{\mathrm{B}} \bar{s}, \\
\sum_{i} e_{i} \sigma_{i z} & =\sum_{q=u, d, s}\left(n_{q_{+}}^{\mathrm{B}} q_{+}+n_{q_{-}}^{\mathrm{B}} q_{-}\right) \\
& =n_{u_{+}}^{\mathrm{B}} u_{+}+n_{u_{-}}^{\mathrm{B}} u_{-}+n_{d_{+}}^{\mathrm{B}} d_{+}+n_{d_{-}}^{\mathrm{B}} d_{-}+n_{s_{+}}^{\mathrm{B}} s_{+}+n_{s_{-}}^{\mathrm{B}} s_{-},
\end{aligned}
$$

where $n_{q}^{\mathrm{B}}\left(n_{\bar{q}}^{\mathrm{B}}\right)$ is the number of quarks with charge $q(\bar{q})$ and $n_{q_{+}}^{\mathrm{B}}\left(n_{q_{-}}^{\mathrm{B}}\right)$ is the number of polarized quarks $q_{+}\left(q_{-}\right)$. For a given baryon $u=-\bar{u}$ and $u_{+}=-u_{-}$, with similar relations for the $d$ and $s$ quarks. The general expression for the charge radii of any of the spin $(1 / 2)^{+}$octet baryon in (4) can be expressed as

$$
\begin{aligned}
\widehat{r_{\mathrm{B}}^{2}}= & (\mathrm{A}-3 \mathrm{~B})\left(\sum_{u, d, s} n_{q}-\sum_{\bar{u}, \bar{d}, \bar{s}} n_{\bar{q}}\right) q \\
& +3(\mathrm{~B}-\mathrm{C})\left(\sum_{u, d, s} n_{q_{+}}-\sum_{u, d, s} n_{q_{-}}\right) q_{+} .
\end{aligned}
$$

Before we discuss the details of the charge radii calculations, it is essential to define the octet and decuplet wavefunctions. The "mixed" state octet baryon wavefunction generated by the spin-spin forces $[33,34]$ which improves the predictions of the various spin-related properties [117-119] is expressed as

$$
|\mathrm{B}\rangle \equiv\left|8, \frac{1}{2}^{+}\right\rangle=\cos \theta\left|56,0^{+}\right\rangle_{N=0}+\sin \theta\left|70,0^{+}\right\rangle_{N=2},
$$

with

$$
\begin{aligned}
\left|56,0^{+}\right\rangle_{N=0}= & \frac{1}{\sqrt{2}}\left(\varphi^{\prime} \chi^{\prime}+\varphi^{\prime \prime} \chi^{\prime \prime}\right) \psi^{s}\left(0^{+}\right), \\
\left|70,0^{+}\right\rangle_{N=2}= & \frac{1}{2}\left[\left(\varphi^{\prime} \chi^{\prime \prime}+\varphi^{\prime \prime} \chi^{\prime}\right) \psi^{\prime}\left(0^{+}\right)\right. \\
& \left.+\left(\varphi^{\prime} \chi^{\prime}-\varphi^{\prime \prime} \chi^{\prime \prime}\right) \psi^{\prime \prime}\left(0^{+}\right)\right] .
\end{aligned}
$$

Here $\theta$ is the mixing angle and $\chi, \varphi$, and $\psi$ are the spin, isospin, and spatial wavefunctions. For the details of the wavefunction, we refer the readers to $[33,34]$. Using the 
TABLE 1: Charge radii of octet baryons in $\mathrm{NQM}_{\text {config }}$ in terms of the GPM parameters. The results in NQM without configuration can easily be calculated by substituting $\theta=0$.

\begin{tabular}{lc}
\hline Charge radii & $\mathrm{NQM}_{\operatorname{config}}$ \\
\hline$r_{p}^{2}$ & $(\mathrm{~A}-3 \mathrm{~B})[2 u+d]+(\mathrm{B}-\mathrm{C})\left[\cos ^{2} \theta\left(4 u_{+}-d_{+}\right)+\sin ^{2} \theta\left(2 u_{+}+d_{+}\right)\right]$ \\
$r_{n}^{2}$ & $(\mathrm{~A}-3 \mathrm{~B})[u+2 d]+(\mathrm{B}-\mathrm{C})\left[\cos ^{2} \theta\left(-u_{+}+4 d_{+}\right)+\sin ^{2} \theta\left(u_{+}+2 d_{+}\right)\right]$ \\
$r_{\Sigma^{+}}^{2}$ & $(\mathrm{~A}-3 \mathrm{~B})[2 u+s]+(\mathrm{B}-\mathrm{C})\left[\cos ^{2} \theta\left(4 u_{+}-s_{+}\right)+\sin ^{2} \theta\left(2 u_{+}+s_{+}\right)\right]$ \\
$r_{\Sigma^{-}}^{2}$ & $-(\mathrm{A}-3 \mathrm{~B})[2 d+s]-(\mathrm{B}-\mathrm{C})\left[\cos ^{2} \theta\left(4 d_{+}-s_{+}\right)+\sin ^{2} \theta\left(2 d_{+}+s_{+}\right)\right]$ \\
$r_{\Sigma^{0}}^{2}$ & $(\mathrm{~A}-3 \mathrm{~B})[u+d+s]+(\mathrm{B}-\mathrm{C})\left[\cos ^{2} \theta\left(2 u_{+}+2 d_{+}-s_{+}\right)+\sin ^{2} \theta\left(u_{+}+d_{+}+s_{+}\right)\right]$ \\
$r_{\Xi^{0}}^{2}$ & $(\mathrm{~A}-3 \mathrm{~B})[u+2 s]+(\mathrm{B}-\mathrm{C})\left[\cos ^{2} \theta\left(-u_{+}+4 s_{+}\right)+\sin ^{2} \theta\left(u_{+}+2 s_{+}\right)\right]$ \\
$r_{\Xi^{-}}^{2}$ & $-(\mathrm{A}-3 \mathrm{~B})[d+2 s]+(\mathrm{B}-\mathrm{C})\left[\cos ^{2} \theta\left(-d_{+}+4 s_{+}\right)+\sin ^{2} \theta\left(d_{+}+2 s_{+}\right)\right]$ \\
$r_{\Lambda}^{2}$ & $(\mathrm{~A}-3 \mathrm{~B})[u+d+s]+(\mathrm{B}-\mathrm{C})\left[\cos ^{2} \theta\left(3 s_{+}\right)+\sin ^{2} \theta\left(u_{+}+d_{+}+s_{+}\right)\right]$ \\
$r_{\Sigma \Lambda}^{2}$ & $(\mathrm{~A}-3 \mathrm{~B})[u+d+s]+\sqrt{3}(\mathrm{~B}-\mathrm{C})\left[u_{+}-d_{+}\right]$ \\
\hline
\end{tabular}

TABLE 2: Charge radii of decuplet baryons in NQM in terms of GPM parameters.

\begin{tabular}{lc}
\hline $\begin{array}{l}\text { Charge } \\
\text { radii }\end{array}$ & $\mathrm{NQM}$ \\
\hline$r_{\Delta^{++}}^{2}$ & $\frac{1}{2}\left[(\mathrm{~A}-3 \mathrm{~B}+6 \mathrm{C})(3 u)+5(\mathrm{~B}-\mathrm{C})\left(3 u_{+}\right)\right]$ \\
$r_{\Delta^{+}}^{2}$ & $(\mathrm{~A}-3 \mathrm{~B}+6 \mathrm{C})(2 u+d)+5(\mathrm{~B}-\mathrm{C})\left(2 u_{+}+d_{+}\right)$ \\
$r_{\Delta^{0}}^{2}$ & $(\mathrm{~A}-3 \mathrm{~B}+6 \mathrm{C})(u+2 d)+5(\mathrm{~B}-\mathrm{C})\left(u_{+}+2 d_{+}\right)$ \\
$r_{\Delta^{-}}^{2}$ & $-(\mathrm{A}-3 \mathrm{~B}+6 \mathrm{C})(3 d)-5(\mathrm{~B}-\mathrm{C})\left(3 d_{+}\right)$ \\
$r_{\Sigma^{*+}}^{2}$ & $(\mathrm{~A}-3 \mathrm{~B}+6 \mathrm{C})(2 u+s)+5(\mathrm{~B}-\mathrm{C})\left(2 u_{+}+s_{+}\right)$ \\
$r_{\Sigma^{*-}}^{2}$ & $-(\mathrm{A}-3 \mathrm{~B}+6 \mathrm{C})(2 d+s)-5(\mathrm{~B}-\mathrm{C})\left(2 d_{+}+s_{+}\right)$ \\
$r_{\Sigma^{* 0}}^{2}$ & $(\mathrm{~A}-3 \mathrm{~B}+6 \mathrm{C})(u+d+s)+5(\mathrm{~B}-\mathrm{C})\left(u_{+}+d_{+}+s_{+}\right)$ \\
$r_{\Xi^{* 0}}^{2}$ & $(\mathrm{~A}-3 \mathrm{~B}+6 \mathrm{C})(u+2 s)+5(\mathrm{~B}-\mathrm{C})\left(u_{+}+2 s_{+}\right)$ \\
$r_{\Xi^{*-}}^{2}$ & $-(\mathrm{A}-3 \mathrm{~B}+6 \mathrm{C})(d+2 s)-5(\mathrm{~B}-\mathrm{C})\left(d_{+}+2 s_{+}\right)$ \\
$r_{\Omega^{-}}^{2}$ & $-(\mathrm{A}-3 \mathrm{~B}+6 \mathrm{C})(3 s)-5(\mathrm{~B}-\mathrm{C})\left(3 s_{+}\right)$ \\
\hline
\end{tabular}

"mixed" wavefunction (15), the charge radii for the $p$ and $\Sigma^{+}$ from (14) can now be expressed as

$$
\begin{aligned}
r_{p}^{2}= & (\mathrm{A}-3 \mathrm{~B})(2 u+d)+3(\mathrm{~B}-\mathrm{C}) \\
& \times\left[\cos ^{2} \theta\left(\frac{4}{3} u_{+}-\frac{1}{3} d_{+}\right)+\sin ^{2} \theta\left(\frac{2}{3} u_{+}+\frac{1}{3} d_{+}\right)\right], \\
r_{\Sigma^{+}}^{2}= & (\mathrm{A}-3 \mathrm{~B})(2 u+s)+3(\mathrm{~B}-\mathrm{C}) \\
& \times\left[\cos ^{2} \theta\left(\frac{4}{3} u_{+}-\frac{1}{3} s_{+}\right)+\sin ^{2} \theta\left(\frac{2}{3} u_{+}+\frac{1}{3} s_{+}\right)\right] .
\end{aligned}
$$

The expressions for the charge radii of other octet baryons in $\mathrm{NQM}$ with configuration mixing $\left(\mathrm{NQM}_{\text {config }}\right)$ are presented in Table 1 . The results without configuration mixing can easily be obtained by taking the mixing angle $\theta=0$.
Configuration mixing generated by the spin-spin forces does not affect the spin $(3 / 2)^{+}$decuplet baryons. The wavefunction in this case is given as

$$
\left|\mathrm{B}^{*}\right\rangle \equiv\left|10, \frac{3}{2}^{+}\right\rangle=\left|56,0^{+}\right\rangle_{N=0}=\chi^{s} \varphi^{s} \psi^{s}\left(0^{+}\right),
$$

Using the baryon wavefunction from the above equation and the charge radii operator from (9), the general expression for the charge radii of spin $(3 / 2)^{+}$baryons can be expressed as

$$
\begin{aligned}
\widehat{r_{\mathrm{B}^{*}}^{2}}= & (\mathrm{A}-3 \mathrm{~B}+6 \mathrm{C})\left(\sum_{u, d, s} n_{q}-\sum_{\bar{u}, \bar{d}, \bar{s}} n_{\bar{q}}\right) q \\
& +5(\mathrm{~B}-\mathrm{C})\left(\sum_{u, d, s} n_{q_{+}}-\sum_{u, d, s} n_{q_{-}}\right) q_{+}
\end{aligned}
$$

As an example, the charge radii for $\Delta^{+}$baryon can be expressed as

$$
r_{\Delta^{+}}^{2}=(\mathrm{A}-3 \mathrm{~B}+6 \mathrm{C})(2 u+d)+5(\mathrm{~B}-\mathrm{C})\left(2 u_{+}+d_{+}\right) .
$$

The expressions for the charge radii of other decuplet baryons in NQM are presented in Table 2.

For the spin $(1 / 2)^{+}$octet baryons, the quadrupole moment of $p$ and $\Sigma^{+}$in NQM can be expressed as

$$
\begin{aligned}
Q_{p}= & 3 \mathrm{~B}^{\prime}\left(2 u+d-2 u_{+}-d_{+}\right) \\
& +\mathrm{C}^{\prime}\left(-4 u+d+4 u_{+}-d_{+}\right), \\
Q_{\Sigma^{+}}= & 3 \mathrm{~B}^{\prime}\left(2 u+s-2 u_{+}-s_{+}\right) \\
& +\mathrm{C}^{\prime}\left(-4 u+s+4 u_{+}-s_{+}\right) .
\end{aligned}
$$


For the spin $(3 / 2)^{+}$decuplet baryons, the quadrupole moment of $\Delta^{+}$and $\Xi^{*-}$ can be expressed as

$$
\begin{aligned}
Q_{\Delta^{+}}= & \mathrm{B}^{\prime}\left(6 u+3 d+2 u_{+}+d_{+}\right) \\
& +\mathrm{C}^{\prime}\left(-6 u-3 d+10 u_{+}+5 d_{+}\right), \\
Q_{\Xi^{*-}}= & \mathrm{B}^{\prime}\left(3 d+6 s+d_{+}+2 s_{+}\right) \\
& +\mathrm{C}^{\prime}\left(-3 d-6 s+5 d_{+}+10 s_{+}\right) .
\end{aligned}
$$

Similarly, for the spin $(3 / 2)^{+} \rightarrow(1 / 2)^{+}$transitions, the quadrupole moment of the $\Delta^{+} p$ and $\Sigma^{*-} \Sigma^{-}$transitions can be expressed as

$$
\begin{gathered}
Q_{\Delta^{+} p}=2 \sqrt{2} \mathrm{~B}^{\prime}\left(u_{+}-d_{+}\right)+2 \sqrt{2} \mathrm{C}^{\prime}(-u+d), \\
Q_{\Sigma^{*-\Sigma^{-}}}=2 \sqrt{2} \mathrm{~B}^{\prime}\left(d_{+}-s_{+}\right)+2 \sqrt{2} \mathrm{C}^{\prime}(-d+s) .
\end{gathered}
$$

The expressions for quadrupole moment of the other $(1 / 2)^{+}$ octet and $(3 / 2)^{+}$decuplet baryons and for $\operatorname{spin}(3 / 2)^{+} \rightarrow$ $(1 / 2)^{+}$transitions in NQM can similarly be calculated. The results are presented in Tables 7,8 , and 9.

\section{Chiral Constituent Quark Model ( $\chi$ CQM)}

In light of the recent developments and successes of the $\chi \mathrm{CQM}$ in explaining the low energy phenomenology [117127], we formulate the quadrupole moments for the $(3 / 2)^{+}$ decuplet baryons and spin $(3 / 2)^{+} \rightarrow(1 / 2)^{+}$transitions. The basic process in the $\chi \mathrm{CQM}$ is the Goldstone boson (GB) emission by a constituent quark which further splits into a $q \bar{q}$ pair as

$$
q_{ \pm} \longrightarrow \mathrm{GB}^{0}+q_{\mp}^{\prime} \longrightarrow\left(q \bar{q}^{\prime}\right)+q_{\mp}^{\prime},
$$

where $q \bar{q}^{\prime}+q^{\prime}$ constitute the "quark sea" [111-116]. The effective Lagrangian describing the interaction between quarks and a nonet of GBs is

$$
\mathscr{L}=g_{8} \bar{q} \Phi^{\prime} q
$$

with

$$
q=\left(\begin{array}{l}
u \\
d \\
s
\end{array}\right)
$$

$$
\Phi^{\prime}=\left(\begin{array}{ccc}
\frac{\pi^{0}}{\sqrt{2}}+\beta \frac{\eta}{\sqrt{6}}+\zeta \frac{\eta^{\prime}}{\sqrt{3}} & \pi^{+} & \alpha K^{+} \\
\pi^{-} & -\frac{\pi^{0}}{\sqrt{2}}+\beta \frac{\eta}{\sqrt{6}}+\zeta \frac{\eta^{\prime}}{\sqrt{3}} & \alpha K^{0} \\
\alpha K^{-} & \alpha \bar{K}^{0} & -\beta \frac{2 \eta}{\sqrt{6}}+\zeta \frac{\eta^{\prime}}{\sqrt{3}}
\end{array}\right),
$$

where $\zeta=g_{1} / g_{8}, g_{1}$ and $g_{8}$ are the coupling constants for the singlet and octet GBs, respectively. If the parameter $a$ (= $\left.\left|g_{8}\right|^{2}\right)$ denotes the transition probability of chiral fluctuation of the splitting $u(d) \rightarrow d(u)+\pi^{+(-)}$, then $\alpha^{2} a, \beta^{2} a$ and $\zeta^{2} a$, respectively, denote the probabilities of transitions of $u(d) \rightarrow$ $s+K^{-(0)}, u(d, s) \rightarrow u(d, s)+\eta$, and $u(d, s) \rightarrow u(d, s)+$ $\eta^{\prime}$. SU(3) symmetry breaking is introduced by considering $M_{s}>M_{u, d}$ as well as by considering the masses of GBs to be nondegenerate $\left(M_{K, \eta}>M_{\pi}\right.$ and $\left.M_{\eta^{\prime}}>M_{K, \eta}\right)$ [111-119].

In terms of the quark contents, the GB field can be expressed as

$$
\Phi^{\prime}=\left(\begin{array}{ccc}
\phi_{u u} u \bar{u}+\phi_{u d} d \bar{d}+\phi_{u s} s \bar{s} & \varphi_{u d} u \bar{d} & \varphi_{u s} u \bar{s} \\
\varphi_{d u} d \bar{u} & \phi_{d u} u \bar{u}+\phi_{d d} d \bar{d}+\phi_{d s} s \bar{s} & \varphi_{d s} d \bar{s} \\
\varphi_{s u} s \bar{u} & \varphi_{s d} s \bar{d} & \phi_{s u} u \bar{u}+\phi_{s d} d \bar{d}+\phi_{s s} s \bar{s}
\end{array}\right)
$$

where

$$
\begin{gathered}
\phi_{u u}=\phi_{d d}=\frac{1}{2}+\frac{\beta}{6}+\frac{\zeta}{3}, \quad \phi_{s s}=\frac{2 \beta}{3}+\frac{\zeta}{3}, \\
\phi_{u s}=\phi_{d s}=\phi_{s u}=\phi_{s d}=-\frac{\beta}{3}+\frac{\zeta}{3}, \\
\phi_{d u}=\phi_{u d}=-\frac{1}{2}+\frac{\beta}{6}+\frac{\zeta}{3}, \quad \varphi_{u d}=\varphi_{d u}=1, \\
\varphi_{u s}=\varphi_{d s}=\varphi_{s u}=\varphi_{s d}=\alpha .
\end{gathered}
$$

A redistribution of flavor and spin structure takes place in the interior of baryon due to the chiral symmetry breaking,

and the modified flavor and spin content of the baryon can be calculated by substituting for every constituent quark:

$$
\begin{gathered}
q \longrightarrow P_{q} q+|\psi(q)|^{2}, \\
q_{ \pm} \longrightarrow P_{q} q_{ \pm}+\left|\psi\left(q_{ \pm}\right)\right|^{2} .
\end{gathered}
$$

Here $P_{q}=1-\sum P_{q}$ is the transition probability of no emission of GB from any of the $q$ quark with

$$
\begin{aligned}
& \sum P_{u}=a\left(\phi_{u u}^{2}+\phi_{u d}^{2}+\phi_{u s}^{2}+\varphi_{u d}^{2}+\varphi_{u s}^{2}\right), \\
& \sum P_{d}=a\left(\phi_{d u}^{2}+\phi_{d d}^{2}+\phi_{d s}^{2}+\varphi_{d u}^{2}+\varphi_{d s}^{2}\right),
\end{aligned}
$$




$$
\sum P_{s}=a\left(\phi_{s u}^{2}+\phi_{s d}^{2}+\phi_{s s}^{2}+\varphi_{s u}^{2}+\varphi_{s d}^{2}\right),
$$

and $|\psi(q)|^{2}\left(\left|\psi\left(q_{ \pm}\right)\right|^{2}\right)$ are the transition probabilities of the emission of $q\left(q_{ \pm}\right)$quark:

$$
\begin{gathered}
|\psi(u)|^{2}=a\left[\left(2 \phi_{u u}^{2}+\phi_{u d}^{2}+\phi_{u s}^{2}+\varphi_{u d}^{2}+\varphi_{u s}^{2}\right) u+\phi_{u u}^{2} \bar{u}\right. \\
\left.+\left(\phi_{u d}^{2}+\varphi_{u d}^{2}\right)(d+\bar{d})+\left(\phi_{u s}^{2}+\varphi_{u s}^{2}\right)(s+\bar{s})\right], \\
|\psi(d)|^{2}=a\left[\left(\phi_{d u}^{2}+2 \phi_{d d}^{2}+\phi_{d s}^{2}+\varphi_{d u}^{2}+\varphi_{d s}^{2}\right) d+\phi_{d d}^{2} \bar{d}\right. \\
\left.+\left(\phi_{d u}^{2}+\varphi_{d u}^{2}\right)(u+\bar{u})+\left(\phi_{u s}^{2}+\varphi_{u s}^{2}\right)(s+\bar{s})\right], \\
|\psi(s)|^{2}=a\left[\left(\phi_{s u}^{2}+\phi_{s d}^{2}+2 \phi_{s s}^{2}+\varphi_{s u}^{2}+\varphi_{s d}^{2}\right) s+\phi_{s s}^{2} \bar{s}\right. \\
\left.+\left(\phi_{s u}^{2}+\varphi_{s u}^{2}\right)(u+\bar{u})+\left(\phi_{s d}^{2}+\varphi_{s d}^{2}\right)(d+\bar{d})\right], \\
\left|\psi\left(u_{ \pm}\right)\right|^{2}=a\left[\left(\phi_{u u}^{2}+\phi_{u d}^{2}+\phi_{u s}^{2}\right) u_{\mp}+\varphi_{u d}^{2} d_{\mp}+\varphi_{u s}^{2} s_{\mp}\right], \\
\left|\psi\left(d_{ \pm}\right)\right|^{2}=a\left[\varphi_{d u}^{2} u_{\mp}+\left(\phi_{d u}^{2}+\phi_{d d}^{2}+\phi_{d s}^{2}\right) d_{\mp}+\varphi_{d s}^{2} s_{\mp}\right], \\
\left|\psi\left(s_{ \pm}\right)\right|^{2}=a\left[\varphi_{s u}^{2} u_{\mp}+\varphi_{s d}^{2} d_{\mp}+\left(\phi_{s u}^{2}+\phi_{s d}^{2}+\phi_{s s}^{2}\right) s_{\mp}\right] .
\end{gathered}
$$

After the inclusion of "quark sea," the charge radii for the spin $(1 / 2)^{+}$octet baryons, in $\chi \mathrm{CQM}$ with configuration mixing ( $\left.\chi \mathrm{CQM}_{\text {config }}\right)$, can be obtained by substituting (29) for each quark in (17). The charge radii for the case of $p$ and $\Sigma^{+}$ are now expressed as

$$
\begin{gathered}
r_{p}^{2}=(\mathrm{A}-3 \mathrm{~B})\left(2 P_{u} u+2|\psi(u)|^{2}+P_{d} d+|\psi(d)|^{2}\right) \\
+3(\mathrm{~B}-\mathrm{C})\left[\operatorname { c o s } ^ { 2 } \theta \left(\frac{4}{3} P_{u} u_{+}+\frac{4}{3}\left|\psi\left(u_{+}\right)\right|^{2}\right.\right. \\
\left.-\frac{1}{3} P_{d} d_{+}-\frac{1}{3}\left|\psi\left(d_{+}\right)\right|^{2}\right) \\
+\sin ^{2} \theta\left(\frac{2}{3} P_{u} u_{+}+\frac{2}{3}\left|\psi\left(u_{+}\right)\right|^{2}\right. \\
\left.\left.+\frac{1}{3} P_{d} d_{+}+\frac{1}{3}\left|\psi\left(d_{+}\right)\right|^{2}\right)\right] \\
r_{\Sigma^{+}}^{2}=(\mathrm{A}-3 \mathrm{~B})\left(2 P_{u} u+2|\psi(u)|^{2}+P_{s} s+|\psi(s)|^{2}\right) \\
+3(\mathrm{~B}-\mathrm{C})\left[\operatorname { c o s } ^ { 2 } \theta \left(\frac{4}{3} P_{u} u_{+}+\frac{4}{3}|\psi(u)|^{2}\right.\right. \\
\left.-\frac{1}{3} P_{s} s_{+}-\frac{1}{3}|\psi(s)|^{2}\right) \\
+\sin ^{2} \theta\left(\frac{2}{3} P_{u} u_{+}+\frac{2}{3}\left|\psi\left(u_{+}\right)\right|^{2}\right. \\
\left.\left.+\frac{1}{3} P_{s} s_{+}+\frac{1}{3}\left|\psi\left(s_{+}\right)\right|^{2}\right)\right] .
\end{gathered}
$$

The charge radii in the $\chi \mathrm{CQM}_{\text {config }}$ for other spin $(1 / 2)^{+}$ octet baryons are presented in Table 3 . The results without configuration mixing can easily be obtained by taking the mixing angle $\theta=0$.

Similarly, for the spin $(3 / 2)^{+}$decuplet baryons, the charge radii are modified on substituting for each quark from (29). For example, the charge radii for $\Delta^{+}$in $\chi \mathrm{CQM}$ can be expressed as

$$
\begin{aligned}
r_{\Delta^{+}}^{2}= & (\mathrm{A}-3 \mathrm{~B}+6 \mathrm{C})\left(2 P_{u} u+2|\psi(u)|^{2}+P_{d} d+|\psi(d)|^{2}\right) \\
& +5(\mathrm{~B}-\mathrm{C})\left(2 P_{u} u_{+}+2\left|\psi\left(u_{+}\right)\right|^{2}+P_{d} d_{+}+\left|\psi\left(d_{+}\right)\right|^{2}\right) .
\end{aligned}
$$

The charge radii of the other decuplet baryons can be calculated similarly and are detailed in Table 4 .

After the inclusion of "quark sea," the quadrupole moment for the spin $(1 / 2)^{+}$octet baryons vanishes on account of the effective cancelation of contribution coming from the "quark sea" and the orbital angular momentum as observed spectroscopically. For the spin $(3 / 2)^{+}$decuplet baryons, the quadrupole moment in $\chi \mathrm{CQM}$ can be obtained by substituting (29) for each quark in (21). The quadrupole moment of $\Delta^{+}$and $\Xi^{*-}$ in $\chi \mathrm{CQM}$ can be expressed as

$$
\begin{gathered}
Q^{\Delta^{+}}=4 \mathrm{~B}^{\prime}+2 \mathrm{C}^{\prime}-\left(\mathrm{B}^{\prime}+5 \mathrm{C}^{\prime}\right) a\left(2+\frac{\beta^{2}}{3}+\frac{2 \zeta^{2}}{3}\right), \\
Q^{\Xi^{*-}}=-4 \mathrm{~B}^{\prime}-2 \mathrm{C}^{\prime}+\left(\mathrm{B}^{\prime}+5 \mathrm{C}^{\prime}\right) a\left(\frac{4 \alpha^{2}}{3}+\beta^{2}+\frac{2 \zeta^{2}}{3}\right) .
\end{gathered}
$$

Similarly, the quadrupole moment of $\Delta^{+} p$ and $\Sigma^{*-} \Sigma^{-}$ transitions in $\chi \mathrm{CQM}$ can be expressed as

$$
\begin{gathered}
Q^{\Delta^{+} p}=2 \sqrt{2}\left[\mathrm{~B}^{\prime}\left(1-a\left(1+\alpha^{2}+\frac{\beta^{2}}{3}+\frac{2 \zeta^{2}}{3}\right)\right)-\mathrm{C}^{\prime}\right], \\
Q^{\Sigma^{*-} \Sigma^{-}}=2 \sqrt{2} \mathrm{~B}^{\prime} a\left(\frac{\alpha^{2}}{3}-\frac{\beta^{2}}{3}\right) .
\end{gathered}
$$

The expressions for the quadrupole moment of other $(3 / 2)^{+}$ decuplet baryons and spin $(3 / 2)^{+} \rightarrow(1 / 2)^{+}$transitions in $\chi \mathrm{CQM}$ can similarly be calculated. The results are presented in Tables 8 and 9.

\section{Results and Discussion}

The calculations of charge radii and quadrupole moment of octet and decuplet baryons involve two set of parameters the $\mathrm{SU}(3)$ symmetry breaking parameters of $\chi \mathrm{CQM}$ and the GPM parameters. The $\chi \mathrm{CQM}$ parameters $a, a \alpha^{2}, a \beta^{2}$, and $a \zeta^{2}$ represent, respectively, the probabilities of fluctuations to pions $K, \eta$, and $\eta^{\prime}$. A best fit of $\chi \mathrm{CQM}$ parameters can be obtained by carrying out a fine grained analysis of the spin and flavor distribution functions [117-119] leading to

$$
a=0.12, \quad \alpha=0.7, \quad \beta=0.4, \quad \zeta=-0.15 \text {. }
$$


TABLE 3: Charge radii of octet baryons in $\chi \mathrm{CQM}_{\text {config }}$ in terms of $\mathrm{SU}(3)$ symmetry breaking parameters and GPM parameters. These results are obtained by substituting $q \rightarrow P_{q} q+|\psi(q)|^{2}$ and $q_{ \pm} \rightarrow P_{q} q_{ \pm}+\left|\psi\left(q_{ \pm}\right)\right|^{2}$ for every constituent quark in NQM. Results in $\chi$ CQM without configuration mixing can easily be obtained by substituting the mixing angle $\theta=0$.

\begin{tabular}{lr}
\hline Charge radii & $\chi \mathrm{CQM}_{\text {config }}$ \\
\hline$r_{p}^{2}$ & $\mathrm{~A}-3 \mathrm{~B}+(\mathrm{B}-\mathrm{C})\left[\cos ^{2} \theta\left(3-a\left(4+2 \alpha^{2}+\beta^{2}+2 \zeta^{2}\right)\right)+\sin ^{2} \theta\left(1-\frac{a}{3}\left(6+\beta^{2}+2 \zeta^{2}\right)\right)\right]$ \\
$r_{n}^{2}$ & $(\mathrm{~B}-\mathrm{C})\left[\cos ^{2} \theta\left(-2+\frac{a}{3}\left(3+9 \alpha^{2}+2 \beta^{2}+4 \zeta^{2}\right)\right)+\sin ^{2} \theta\left(a\left(-1+\alpha^{2}\right)\right)\right]$ \\
\hline$r_{\Sigma^{+}}^{2}$ & $\mathrm{~A}-3 \mathrm{~B}+(\mathrm{B}-\mathrm{C})\left[\cos ^{2} \theta\left(3-\frac{a}{3}\left(12+5 \alpha^{2}+4 \beta^{2}+6 \zeta^{2}\right)\right)+\sin ^{2} \theta\left(1-\frac{a}{3}\left(6+\alpha^{2}+2 \zeta^{2}\right)\right)\right]$ \\
$r_{\Sigma^{-}}^{2}$ & $\mathrm{~A}-3 \mathrm{~B}+(\mathrm{B}-\mathrm{C})\left[\cos ^{2} \theta\left(-1+\frac{a}{3}\left(7 \alpha^{2}+2 \zeta^{2}\right)\right)+\sin ^{2} \theta\left(-1+\frac{a}{3}\left(5 \alpha^{2}+2 \beta^{2}+2 \zeta^{2}\right)\right)\right]$ \\
$r_{\Sigma^{0}}^{2}$ & $(\mathrm{~B}-\mathrm{C})\left[\cos ^{2} \theta\left(1-\frac{a}{3}\left(6-\alpha^{2}+2 \beta^{2}+2 \zeta^{2}\right)\right)+\sin ^{2} \theta\left(\frac{a}{3}\left(-3+2 \alpha^{2}+\beta^{2}\right)\right)\right]$ \\
\hline$r_{\Xi^{0}}^{2}$ & $(\mathrm{~B}-\mathrm{C})\left[\cos ^{2} \theta\left(-2+\frac{a}{3}\left(3+5 \alpha^{2}+6 \beta^{2}+4 \zeta^{2}\right)\right)+\sin ^{2} \theta\left(\frac{a}{3}\left(-3+\alpha^{2}+2 \beta^{2}\right)\right)\right]$ \\
$r_{\Xi^{-}}^{2}$ & $\mathrm{~A}-3 \mathrm{~B}+(\mathrm{B}-\mathrm{C})\left[\cos ^{2} \theta\left(-1+\frac{a}{3}\left(2 \alpha^{2}+5 \beta^{2}+2 \zeta^{2}\right)\right)+\sin ^{2} \theta\left(-1+\frac{a}{3}\left(4 \alpha^{2}+3 \beta^{2}+2 \zeta^{2}\right)\right)\right]$ \\
$r_{\Lambda}^{2}$ & $(\mathrm{~B}-\mathrm{C})\left[\cos ^{2} \theta\left(-1+\frac{a}{3}\left(3 \alpha^{2}+4 \beta^{2}+2 \zeta^{2}\right)\right)+\sin ^{2} \theta\left(\frac{a}{9}\left(-9+6 \alpha^{2}+7 \beta^{2}+2 \zeta^{2}\right)\right)\right]$ \\
$r_{\Sigma \Lambda}^{2}$ & $(\mathrm{~B}-\mathrm{C})\left[\sqrt{3}-\frac{a}{\sqrt{3}}\left(3+3 \alpha^{2}+\beta^{2}+2 \zeta^{2}\right)\right]$ \\
\hline
\end{tabular}

TABLE 4: Charge radii of decuplet baryons in $\chi \mathrm{CQM}$ in terms of $\mathrm{SU}(3)$ symmetry breaking parameters and GPM parameters. These results are obtained by substituting $q \rightarrow P_{q} q+|\psi(q)|^{2}$ and $q_{ \pm} \rightarrow$ $P_{q} q_{ \pm}+\left|\psi\left(q_{ \pm}\right)\right|^{2}$ for every constituent quark in NQM.

\begin{tabular}{lc}
\hline Charge radii & $\chi \mathrm{CQM}$ \\
\hline$r_{\Delta^{++}}^{2}$ & $\mathrm{~A}+2 \mathrm{~B}+\mathrm{C}-\frac{5 a}{6}(\mathrm{~B}-\mathrm{C})\left(9+3 \alpha^{2}+2 \beta^{2}+4 \zeta^{2}\right)$ \\
$r_{\Delta^{+}}^{2}$ & $\mathrm{~A}+2 \mathrm{~B}+\mathrm{C}-\frac{5 a}{3}(\mathrm{~B}-\mathrm{C})\left(6+\beta^{2}+2 \zeta^{2}\right)$ \\
$r_{\Delta^{0}}^{2}$ & $5 a(\mathrm{~B}-\mathrm{C})\left(-1+\alpha^{2}\right)$ \\
$r_{\Delta^{-}}^{2}$ & $\mathrm{~A}+2 \mathrm{~B}+\mathrm{C}-\frac{5 a}{3}(\mathrm{~B}-\mathrm{C})\left(6 \alpha^{2}+\beta^{2}+2 \zeta^{2}\right)$ \\
$r_{\Sigma^{*+}}^{2}$ & $\mathrm{~A}+2 \mathrm{~B}+\mathrm{C}-\frac{5 a}{3}(\mathrm{~B}-\mathrm{C})\left(6+\alpha^{2}+2 \zeta^{2}\right)$ \\
$r_{\Sigma^{*-}}^{2}$ & $\mathrm{~A}+2 \mathrm{~B}+\mathrm{C}-\frac{5 a}{3}(\mathrm{~B}-\mathrm{C})\left(5 \alpha^{2}+2 \beta^{2}+2 \zeta^{2}\right)$ \\
$r_{\Sigma^{* 0}}^{2}$ & $\frac{5 a}{3}(\mathrm{~B}-\mathrm{C})\left(-3+2 \alpha^{2}+\beta^{2}\right)$ \\
$r_{\Xi^{* 0}}^{2}$ & $\frac{5 a}{3}(\mathrm{~B}-\mathrm{C})\left(-3+\alpha^{2}+2 \beta^{2}\right)$ \\
$r_{\Xi^{*-}}^{2}$ & $\mathrm{~A}+2 \mathrm{~B}+\mathrm{C}-\frac{5 a}{3}(\mathrm{~B}-\mathrm{C})\left(4 \alpha^{2}+3 \beta^{2}+2 \zeta^{2}\right)$ \\
$r_{\Omega^{-}}^{2}$ & $\mathrm{~A}+2 \mathrm{~B}+\mathrm{C}-\frac{5 a}{3}(\mathrm{~B}-\mathrm{C})\left(3 \alpha^{2}+4 \beta^{2}+2 \zeta^{2}\right)$ \\
\hline
\end{tabular}

The mixing angle $\theta$ is fixed from the consideration of neutron charge radius [28]. This set of parameters has already been tested for a wide variety of low energy matrix elements and has been able to give a simultaneous fit to the quantities describing proton spin and flavor structure [117-119], weak vector-axial vector form factors $[120,121]$, strangeness content in the nucleon [122-124], octet and decuplet baryons magnetic moments [125-127], and so forth.

The order of GPM parameters corresponding to the one-, two-, and three-quark terms decreases with the increasing complexity of terms and obeys the hierarchy $\mathrm{A}>\mathrm{B}>\mathrm{C}$ $[143,144]$. These are fitted by using the available experimental values for the charge radii and quadrupole moment of nucleon as input. In the present case, we have used $r_{p}=$ $0.877 \pm 0.007 \mathrm{fm}[12], r_{n}^{2}=-0.1161 \pm 0.0022 \mathrm{fm}^{2}$ [12], and $Q_{\Delta^{+} N}=-0.0846 \pm 0.0033 \mathrm{fm}^{2}$ [19]. The set of GPM parameters obtained after $\chi^{2}$ minimization are as follows:

$$
\mathrm{A}=0.879, \quad \mathrm{~B}=0.094, \quad \mathrm{C}=0.016 \text {. }
$$

For the quadrupole moment calculations, best fit set of parameters obtained after $\chi^{2}$ minimization are as follows:

$$
\mathrm{B}^{\prime}=-0.047, \quad \mathrm{C}^{\prime}=-0.008,
$$

obeying the hierarchy $\mathrm{B}^{\prime}>\mathrm{C}^{\prime}[143,144]$ corresponding to the two- and three-quark contribution. Since we also intend to investigate the extent to which the three-quark term contributes, we calculate the charge radii corresponding to the one- and two-quark terms only by taking $\mathrm{C}=0$. Similarly, if we intend to calculate the charge radii corresponding to just the one-quark term, we can take $\mathrm{B}=\mathrm{C}=0$.

Using the set of parameters discussed above, we have calculated the numerical values for the charge radii of octet and decuplet baryons in $\chi \mathrm{CQM}_{\text {config }}$ and presented the results in Tables 5 and 6, respectively. To understand the implications of chiral symmetry breaking and "quark sea," we have also presented the results of NQM as well as comparing our results with the predictions of other available phenomenological models. Since the calculations in $\chi \mathrm{CQM}$ have been carried 
TABLE 5: Charge radii of octet baryons calculated in $\chi$ CQM in comparison with other phenomenological models (in units of fm ${ }^{2}$ ).

\begin{tabular}{|c|c|c|c|c|c|c|c|c|c|c|c|}
\hline \multirow[b]{2}{*}{ Charge radii } & \multirow[b]{2}{*}{ NQM } & \multirow{2}{*}{$\begin{array}{c}\text { RCQM } \\
{[51]}\end{array}$} & \multirow{2}{*}{$\begin{array}{c}\mathrm{HB} \chi \mathrm{PT} \\
{[60]}\end{array}$} & \multirow[b]{2}{*}{$\chi \mathrm{PT}[61,62]$} & \multirow[b]{2}{*}{$\begin{array}{c}\text { CCQM } \\
{[52]}\end{array}$} & \multirow[b]{2}{*}{$\mathrm{P} \chi \mathrm{QM}[59]$} & \multirow[b]{2}{*}{$\begin{array}{l}1 / N_{c} \\
{[57,58]}\end{array}$} & \multirow{2}{*}{$\begin{array}{l}\text { Lattice } \\
{[63]}\end{array}$} & \multirow{2}{*}{$\begin{array}{l}\text { With SU(3) } \\
\text { symmetry }\end{array}$} & \multicolumn{2}{|l|}{$\begin{array}{c}\chi \mathrm{CQM}_{\text {config }} \\
\text { With } \mathrm{SU}(3) \text { sym }\end{array}$} \\
\hline & & & & & & & & & & $\begin{array}{c}\mathrm{A}=0.879 \\
\mathrm{~B}=0.094 \\
\mathrm{C}=0.0\end{array}$ & $\begin{array}{l}A=0.879 \\
B=0.094 \\
C=0.016\end{array}$ \\
\hline$r_{p}^{2}$ & 0.813 & - & 0.735 & 0.717 & 0.82 & $0.72 \pm 0.09$ & 0.779 & 0.685 & 0.732 & 0.801 & 0.766 \\
\hline $\begin{array}{l}r_{p}= \\
0.877 \pm 0.007\end{array}$ & & & & & & & & & & & \\
\hline $\begin{array}{l}r_{n}^{2} \\
-0.1161 \pm 0.0022\end{array}$ & -0.138 & - & -0.113 & -0.113 & -0.13 & $-0.111 \pm 0.014$ & -0.116 & -0.158 & -0.087 & -0.140 & -0.116 \\
\hline$r_{\Sigma^{+}}^{2}$ & 0.813 & 0.79 & 1.366 & $0.60 \pm 0.02$ & 1.13 & $0.81 \pm 0.10$ & 0.928 & 0.749 & 0.732 & 0.802 & 0.767 \\
\hline $\begin{array}{l}r_{\Sigma^{-}}^{2} \\
0.61 \pm 0.21[13]\end{array}$ & 0.675 & 0.49 & 0.798 & $0.67 \pm 0.03$ & 0.72 & $0.71 \pm 0.07$ & 0.672 & 0.657 & 0.646 & 0.678 & 0.664 \\
\hline$r_{\Sigma^{0}}^{2}$ & 0.069 & 0.15 & - & $-0.03 \pm 0.01$ & 0.20 & $0.05 \pm 0.01$ & 0.128 & - & 0.043 & 0.062 & 0.052 \\
\hline$r_{\Xi^{0}}^{2}$ & -0.138 & 0.14 & -0.122 & $0.13 \pm 0.03$ & -0.19 & $0.14 \pm 0.02$ & 0.132 & -0.082 & -0.087 & -0.145 & -0.120 \\
\hline$r_{\Xi^{-}}^{2}$ & 0.675 & 0.47 & 0.997 & $0.49 \pm 0.05$ & 0.54 & $0.62 \pm 0.07$ & 0.520 & 0.502 & 0.646 & 0.683 & 0.669 \\
\hline$r_{\Lambda}^{2}$ & -0.069 & 0.038 & -0.284 & $0.11 \pm 0.02$ & 0.03 & $0.05 \pm 0.01$ & 0.050 & 0.010 & -0.042 & -0.076 & -0.063 \\
\hline$r_{\Sigma \Lambda}^{2}$ & 0.135 & -0.12 & 0.074 & $0.03 \pm 0.01$ & - & 0.0 & -0.066 & - & 0.085 & 0.132 & 0.109 \\
\hline
\end{tabular}

TABLE 6: Charge radii of decuplet baryons calculated in $\chi C Q M$ in comparison with other phenomenological models (in units of fm ${ }^{2}$ ).

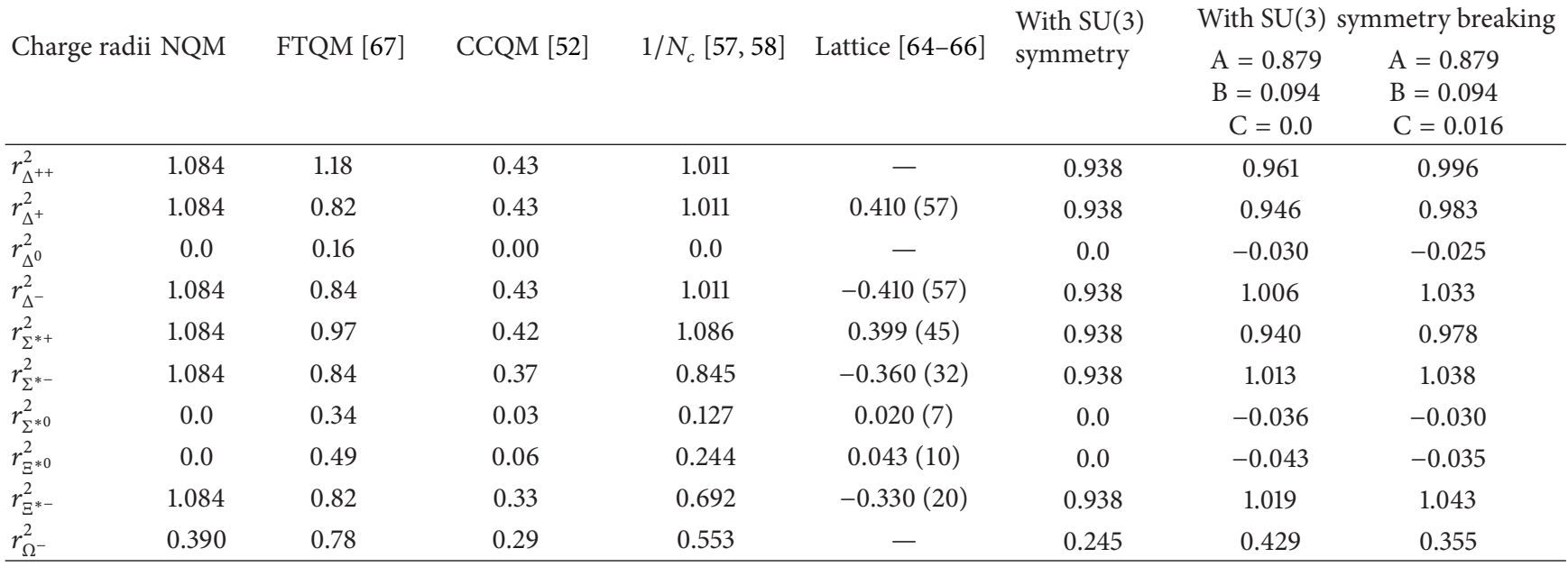

out using the GPM, the NQM results have also been presented by including the one-, two-, and three-quark contributions of the GPM parameters. It is clear from Tables 1 and 2 that if we consider the contribution coming from onequark term only, the charge radii of the charged baryons are equal whereas all neutral baryons have zero charge radii. These predictions are modified on the inclusion of two- and three-quark terms of GPM and are further modified on the inclusion of "quark sea" and SU(3) symmetry breaking effects. Thus, it seems that the GPM parameters alone are able to explain the experimentally observed nonzero charge radii of the neutral baryons. However, NQM is unable to account for the "proton spin problem" and other related quantities; the results have been presented for $\chi \mathrm{CQM}$. The importance of strange quark mass has been investigated by comparing the $\chi \mathrm{CQM}$ results with and without SU(3) symmetry breaking. The SU(3) symmetry results can be easily derived from Tables 3 and 4 by considering $\alpha=\beta=1$ and $\zeta=-1$. The SU(3) breaking results are in general higher in magnitude than the $\mathrm{SU}(3)$ symmetric results, and the values obtained are also in agreement with the other models.

For the case of octet baryons, it can be easily shown from Table 5 that in the SU(3) symmetric limit, octet baryon charge radii can be expressed in terms of the nucleon charge radii leading to the following relations:

$$
r_{\Sigma^{+}}^{2}=r_{p}^{2}, \quad r_{\Xi^{-}}^{2}=r_{\Sigma^{-}}^{2}=r_{p}^{2}+r_{n}^{2},
$$


TABLE 7: Quadrupole moments of the octet baryons in NQM using the GPM.

\begin{tabular}{ll}
\hline Baryons & NQM \\
\hline$p$ & $3 \mathrm{~B}^{\prime}\left(2 u+d-2 u_{+}-d_{+}\right)+\mathrm{C}^{\prime}\left(-4 u+d+4 u_{+}-d_{+}\right)$ \\
$n$ & $3 \mathrm{~B}^{\prime}\left(u+2 d-u_{+}-2 d_{+}\right)+\mathrm{C}^{\prime}\left(u-4 d-u_{+}+4 d_{+}\right)$ \\
$\Sigma^{+}$ & $3 \mathrm{~B}^{\prime}\left(2 u+s-2 u_{+}-s_{+}\right)+\mathrm{C}^{\prime}\left(-4 u+s+4 u_{+}-s_{+}\right)$ \\
$\Sigma^{-}$ & $3 \mathrm{~B}^{\prime}\left(2 d+s-2 d_{+}-s_{+}\right)+\mathrm{C}^{\prime}\left(-4 d+s+4 d_{+}-s_{+}\right)$ \\
$\Sigma^{0}$ & $3 \mathrm{~B}^{\prime}\left(u+d+s-u_{+}-d_{+}-s_{+}\right)$ \\
& $\quad+\mathrm{C}^{\prime}\left(-2 u-2 d+s+2 u_{+}+2 d_{+}-s_{+}\right)$ \\
$\Xi^{0}$ & $3 \mathrm{~B}^{\prime}\left(u+2 s-u_{+}-2 s_{+}\right)+\mathrm{C}^{\prime}\left(u-4 s-u_{+}+4 s_{+}\right)$ \\
$\Xi^{-}$ & $3 \mathrm{~B}^{\prime}\left(d+2 s-d_{+}-2 s_{+}\right)+\mathrm{C}^{\prime}\left(d-4 s-d_{+}+4 s_{+}\right)$ \\
$\Lambda^{0}$ & $3 \mathrm{~B}^{\prime}\left(u+d+s-u_{+}-d_{+}-s_{+}\right)+3 \mathrm{C}^{\prime}\left(-s+s_{+}\right)$ \\
\hline
\end{tabular}

The inclusion of SU(3) symmetry breaking changes this pattern considerably, and we get

$$
r_{\Sigma^{+}}^{2}>r_{p}^{2}, \quad r_{\Xi^{-}}^{2}>r_{\Sigma^{-}}^{2}>r_{p}^{2}+r_{n}^{2} .
$$

Also we have

$$
2 r_{\Lambda}^{2}=-2 r_{\Sigma^{0}}^{2}=r_{\Xi^{0}}^{2}=r_{n}^{2}
$$

which has its importance in the isospin limit where the threequark core in neutral baryons does not contribute to the charge radii. In the limit of $\mathrm{SU}(3)$ symmetry breaking, a nonvanishing value for the neutral baryons charge radii is generated by the "quark sea" through the chiral fluctuations of constituent quarks leading to

$$
r_{\Lambda}^{2}>-r_{\Sigma^{0}}^{2}, \quad r_{\Xi^{0}}^{2}>r_{n}^{2}
$$

The exact order of SU(3) symmetry breaking effects can be easily found from Table 3 . Since experimental information is not available for some of these octet charge radii, the accuracy of these relations can be tested by the future experiments. It is interesting to note that the relation for the $\Sigma$ baryon charge radii,

$$
r_{\Sigma^{+}}^{2}-2 r_{\Sigma^{0}}^{2}-r_{\Sigma^{-}}^{2}=0
$$

holds good even after incorporating SU(3) symmetry breaking. Since this relation is independent of $\mathrm{SU}(3)$ symmetry breaking parameters, any refinement in the $\Sigma$ baryon charge radii data would have important implications for $\mathrm{SU}(3)$ symmetry breaking. Further, our predicted value $r_{\Sigma^{-}}^{2}=$ 0.664 is clearly of the order of proton charge radius and is also in agreement with the recent SELEX collaboration experimental results [12]. It would be important to mention here that the $\chi \mathrm{CQM}$ parameters play an important role in the $\mathrm{SU}(3)$ symmetry breaking effects whereas the assumed parametrization plays a dominant role in the valence quark distributions.

In Table 3, we have presented the results for the case with configuration mixing generated by the spin-spin forces. We have not presented the results without configuration mixing which can easily be obtained by taking the mixing angle $\theta=$ 0 . It has been observed that configuration mixing decreases the overall magnitudes of the charge radii in $\chi \mathrm{CQM}$, but the change is very small as compared to the other low energy properties like spin distribution function, magnetic moments, and so forth [117-127].

On comparing our results with the other phenomenological models, we find that for the case of charged octet baryons, our results are in fair agreement in sign and magnitude with the other model predictions. However, for the neutral octet baryons $n, \Sigma^{0}, \Xi^{0}$, and $\Lambda$, different models show opposite sign. For example, if we consider the charge radii for the $\Lambda$ baryon, our model prediction $(-0.063)$ is opposite in sign to the predictions of the relativistic constituent quark model (RCQM) [51], covariant constituent quark model (CCQM) [52], $1 / N_{c}$ expansion [55, 56], and $\mathrm{P} \chi \mathrm{QM}[59]$. On the other hand, it is in agreement with the sign of $\mathrm{HB} \chi \mathrm{PT}[60]$. A similar trend has been observed for the charge radii of $\Sigma \Lambda$ transition. The difference in the sign may be due to the chiral fluctuation of a constituent quark leading to the reversal of sign in case of neutral octet baryons. This can perhaps be substantiated by a measurement of charge radii of other baryons.

The spin $(3 / 2)^{+}$decuplet baryon charge radii, presented in Table 6, are in general higher than the octet baryon charge radii which are in line with the trend followed by the octet and decuplet baryons for the other low energy hadronic matrix elements such as magnetic moments. In this case also, the inclusion of SU(3) symmetry breaking increases the predictions of charge radii. It can be easily shown that $\mathrm{SU}(3)$ symmetry results in the following relations for the decuplet baryons:

$$
r_{\Delta^{++}}^{2}=r_{\Delta^{+}}^{2}=r_{\Delta^{-}}^{2}=r_{\Sigma^{*+}}^{2}=r_{\Sigma^{*-}}^{2}=r_{\Xi^{*-}}^{2} .
$$

These results are affected by the inclusion of SU(3) symmetry breaking and give

$$
r_{\Xi^{*-}}^{2}>r_{\Sigma^{*-}}^{2}>r_{\Delta^{-}}^{2}>r_{\Delta^{++}}^{2}>r_{\Delta^{+}}^{2}>r_{\Sigma^{*+}}^{2}
$$

Some relations, derived in $1 / N_{c}$ expansion of QCD [55-58], are found to be independent of $\mathrm{SU}(3)$ symmetry breaking parameters in $\chi \mathrm{CQM}$. Even though the individual charge radii are affected by $\mathrm{SU}(3)$ symmetry breaking, the effects are canceled exactly for the following relations:

$$
\begin{gathered}
2 r_{\Delta^{++}}^{2}-r_{\Delta^{+}}^{2}-r_{\Delta^{0}}^{2}-r_{\Delta^{-}}^{2}=0, \\
2 r_{\Delta^{++}}^{2}-3 r_{\Delta^{+}}^{2}+3 r_{\Delta^{0}}^{2}+r_{\Delta^{-}}^{2}=0, \\
r_{\Sigma^{*+}}^{2}-2 r_{\Sigma^{* 0}}^{2}-r_{\Sigma^{*-}}^{2}=0 .
\end{gathered}
$$

In this case also, $\mathrm{SU}(3)$ symmetry breaking is expected to reduce the charge radii with increasing strangeness content. As a consequence, $\Delta^{-}, \Sigma^{-}$, and $\Xi^{-}$should have successively decreasing charge radii. However, this suppression disappears in $\chi \mathrm{CQM}$ due to the effect of "quark sea," and the charge radii of $\Delta^{+}, \Sigma^{*-}$, and $\Xi^{* 0}$ are of almost the same order as that of $\Sigma^{*+}, \Xi^{*-}$, and $\Sigma^{* 0}$, respectively. Again, the sign and magnitude of the decuplet baryon charge radii in $\chi \mathrm{CQM}$ are in fair agreement with the other phenomenological models with the exception for neutral baryons. One of the important predictions in $\chi \mathrm{CQM}$ is a nonzero $\Delta^{0}$ charge radii which 
TABLE 8: Quadrupole moments of the decuplet baryons in NQM and $\chi$ CQM using the GPM.

\begin{tabular}{lll}
\hline Baryon & $\mathrm{NQM}$ & $\chi \mathrm{CQM}$ \\
\hline$\Delta^{++}$ & $\mathrm{B}^{\prime}\left(9 u+3 u_{+}\right)+\mathrm{C}^{\prime}\left(-9 u+15 u_{+}\right)$ & $8 \mathrm{~B}^{\prime}+4 \mathrm{C}^{\prime}-\left(\mathrm{B}^{\prime}+5 \mathrm{C}^{\prime}\right) \frac{a}{3}\left(9+3 \alpha^{2}+2 \beta^{2}+4 \zeta^{2}\right)$ \\
$\Delta^{+}$ & $\mathrm{B}^{\prime}\left(3(2 u+d)+2 u_{+}+d_{+}\right)+\mathrm{C}^{\prime}\left(-3(2 u+d)+5\left(2 u_{+}+d_{+}\right)\right)$ & $4 \mathrm{~B}^{\prime}+2 \mathrm{C}^{\prime}-\left(\mathrm{B}^{\prime}+5 \mathrm{C}^{\prime}\right) \frac{a}{3}\left(6+\beta^{2}+2 \zeta^{2}\right)$ \\
$\Delta^{0}$ & $\mathrm{~B}^{\prime}\left(3(u+2 d)+u_{+}+2 d_{+}\right)+\mathrm{C}^{\prime}\left(-3(u+2 d)+5\left(u_{+}+2 d_{+}\right)\right)$ & $\left(\mathrm{B}^{\prime}+5 \mathrm{C}^{\prime}\right) a\left(-1+\alpha^{2}\right)$ \\
$\Delta^{-}$ & $\mathrm{B}^{\prime}\left(9 d+3 d_{+}\right)+\mathrm{C}^{\prime}\left(-9 d+15 d_{+}\right)$ & $-4 \mathrm{~B}^{\prime}-2 \mathrm{C}^{\prime}+\left(\mathrm{B}^{\prime}+5 \mathrm{C}^{\prime}\right) \frac{a}{3}\left(6 \alpha^{2}+\beta^{2}+2 \zeta^{2}\right)$ \\
$\Sigma^{*+}$ & $\mathrm{B}^{\prime}\left(3(2 u+s)+2 u_{+}+s_{+}\right)+\mathrm{C}^{\prime}\left(-3(2 u+s)+5\left(2 u_{+}+s_{+}\right)\right)$ & $4 \mathrm{~B}^{\prime}+2 \mathrm{C}^{\prime}-\left(\mathrm{B}^{\prime}+5 \mathrm{C}^{\prime}\right) \frac{a}{3}\left(6+\alpha^{2}+2 \zeta^{2}\right)$ \\
$\Sigma^{*-}$ & $\mathrm{B}^{\prime}\left(3(2 d+s)+2 d_{+}+s_{+}\right)+\mathrm{C}^{\prime}\left(-3(2 d+s)+5\left(2 d_{+}+s_{+}\right)\right)$ & $-4 \mathrm{~B}^{\prime}-2 \mathrm{C}^{\prime}+\left(\mathrm{B}^{\prime}+5 \mathrm{C}^{\prime}\right) \frac{a}{3}\left(5 \alpha^{2}+2 \beta^{2}+2 \zeta^{2}\right)$ \\
$\Sigma^{* 0}$ & $\mathrm{~B}^{\prime}\left(3(u+d+s)+u_{+}+d_{+}+s_{+}\right)$ & $\left(\mathrm{B}^{\prime}+5 \mathrm{C}^{\prime}\right) \frac{a}{3}\left(-3+2 \alpha^{2}+\beta^{2}\right)$ \\
$\Xi^{* 0}$ & $\mathrm{~B}^{\prime}\left(3(u+2 s)+u_{+}+2 s_{+}\right)+\mathrm{C}^{\prime}\left(-3(u+2 s)+5\left(u_{+}+2 s_{+}\right)\right)$ & $\left(\mathrm{B}^{\prime}+5 \mathrm{C}^{\prime}\right) \frac{a}{3}\left(-3+\alpha^{2}+2 \beta^{2}\right)$ \\
$\Xi^{*-}$ & $\mathrm{B}^{\prime}\left(3(d+2 s)+d_{+}+2 s_{+}\right)+\mathrm{C}^{\prime}\left(-3(d+2 s)+5\left(d_{+}+2 s_{+}\right)\right)$ & $-4 \mathrm{~B}^{\prime}-2 \mathrm{C}^{\prime}+\left(\mathrm{B}^{\prime}+5 \mathrm{C}^{\prime}\right) \frac{a}{3}\left(4 \alpha^{2}+3 \beta^{2}+2 \zeta^{2}\right)$ \\
$\Omega^{-}$ & $\mathrm{B}^{\prime}\left(9 s+3 s_{+}\right)+\mathrm{C}^{\prime}\left(-9 s+15 s_{+}\right)$ & $-4 \mathrm{~B}^{\prime}-2 \mathrm{C}^{\prime}+\left(\mathrm{B}^{\prime}+5 \mathrm{C}^{\prime}\right) \frac{a}{3}\left(3 \alpha^{2}+4 \beta^{2}+2 \zeta^{2}\right)$ \\
\hline
\end{tabular}

TABLE 9: Quadrupole moments of the spin $(3 / 2)^{+} \rightarrow(1 / 2)^{+}$transitions in NQM and $\chi C Q M$ using the GPM.

\begin{tabular}{lll}
\hline Baryon & NQM & $\chi \mathrm{CQM}$ \\
\hline$\Delta^{+} p$ & $2 \sqrt{2} \mathrm{~B}^{\prime}\left(u_{+}-d_{+}\right)+2 \sqrt{2} \mathrm{C}^{\prime}(-u+d)$ & $2 \sqrt{2} \mathrm{~B}^{\prime}\left(1-\frac{a}{3}\left(3+3 \alpha^{2}+\beta^{2}+2 \zeta^{2}\right)\right)-2 \sqrt{2} \mathrm{C}^{\prime}$ \\
$\Sigma^{*+} \Sigma^{+}$ & $2 \sqrt{2} \mathrm{~B}^{\prime}\left(u_{+}-s_{+}\right)+2 \sqrt{2} \mathrm{C}^{\prime}(-u+s)$ & $2 \sqrt{2} \mathrm{~B}^{\prime}\left(1-\frac{a}{3}\left(3+2 \alpha^{2}+2 \beta^{2}+2 \zeta^{2}\right)\right)-2 \sqrt{2} \mathrm{C}^{\prime}$ \\
$\Sigma^{*-} \Sigma^{-}$ & $2 \sqrt{2} \mathrm{~B}^{\prime}\left(d_{+}-s_{+}\right)+2 \sqrt{2} \mathrm{C}^{\prime}(-d+s)$ & $\frac{2 \sqrt{2}}{3} \mathrm{~B}^{\prime} a\left(\alpha^{2}-\beta^{2}\right)$ \\
$\Sigma^{* 0} \Sigma^{0}$ & $\sqrt{2} \mathrm{~B}^{\prime}\left(u_{+}+d_{+}-2 s_{+}\right)+\sqrt{2} \mathrm{C}^{\prime}(-u-d+2 s)$ & $\sqrt{2} \mathrm{~B}^{\prime}\left(1-a\left(1+\frac{\alpha^{2}}{3}+\beta^{2}+\frac{2}{3} \zeta^{2}\right)\right)-\sqrt{2} \mathrm{C}^{\prime}$ \\
$\Xi^{* 0} \Xi^{0}$ & $2 \sqrt{2} \mathrm{~B}^{\prime}\left(u_{+}-s_{+}\right)+2 \sqrt{2} \mathrm{C}^{\prime}(-u+s)$ & $2 \sqrt{2} \mathrm{~B}^{\prime}\left(1-\frac{a}{3}\left(3+2 \alpha^{2}+2 \beta^{2}+2 \zeta^{2}\right)\right)-2 \sqrt{2} \mathrm{C}^{\prime}$ \\
$\Xi^{*-} \Xi^{-}$ & $2 \sqrt{2} \mathrm{~B}^{\prime}\left(d_{+}-s_{+}\right)+2 \sqrt{2} \mathrm{C}^{\prime}(-d+s)$ & $\frac{2 \sqrt{2}}{3} \mathrm{~B}^{\prime} a\left(\alpha^{2}-\beta^{2}\right)$ \\
$\Sigma^{* 0} \Lambda$ & $\sqrt{6} \mathrm{~B}^{\prime}\left(u_{+}-d_{+}\right)+\sqrt{6} \mathrm{C}^{\prime}(-u+d)$ & $\sqrt{6} \mathrm{~B}^{\prime}\left(1-\frac{a}{3}\left(3+3 \alpha^{2}+\beta^{2}+2 \zeta^{2}\right)\right)-\sqrt{6} \mathrm{C}^{\prime}$ \\
\hline
\end{tabular}

vanishes in NQM as well as in some other models. This is further endorsed by the predictions of the field theoretical quark model (FTQM) calculations [67]. The contribution of the three-quark term in the case of decuplet baryons is exactly opposite to that for the octet baryons. Unlike the octet baryon case, the inclusion of the three-quark term increases the value of the baryon charge radii.

For the sake of completeness, certain relations between the octet and decuplet baryon charge radii can also be tested for the spacing between the levels. In NQM, we have

$$
r_{\Sigma^{-}}^{2}-r_{\Sigma^{*-}}^{2}=r_{\Xi^{-}}^{2}-r_{\Xi^{*-}}^{2}=r_{\Sigma^{+}}^{2}-r_{\Sigma^{*+}}^{2}=r_{\Xi^{0}}^{2}-r_{\Xi^{* 0}}^{2}=r_{n}^{2}
$$

In $\chi \mathrm{CQM}$, the inclusion of $\mathrm{SU}(3)$ symmetry breaking effects creates a spacing between the octet and decuplet baryon charge radii as

$$
r_{p}^{2}-r_{\Delta^{+}}^{2}=r_{\Sigma^{+}}^{2}-r_{\Sigma^{*+}}^{2}=-0.31
$$

$$
\begin{gathered}
r_{\Sigma^{-}}^{2}-r_{\Sigma^{*-}}^{2}=r_{\Xi^{-}}^{2}-r_{\Xi^{*-}}^{2}=-0.48 \\
r_{n}^{2}-r_{\Delta^{0}}^{2}=r_{\Xi^{0}}^{2}-r_{\Xi^{* 0}}^{2}=-0.09 .
\end{gathered}
$$

We have calculated the numerical values for the quadrupole moment for the $(3 / 2)^{+}$decuplet baryons in $\chi \mathrm{CQM}$ and presented the results in Table 10 . The results of the spin $(3 / 2)^{+} \rightarrow(1 / 2)^{+}$transitions have been presented in Table 11. To understand the implications of chiral symmetry breaking and "quark sea," we have also presented the results of NQM. Since the calculations in $\chi C Q M$ have been carried out using the GPM, the NQM results have also been presented by including the two- and three- quark term contributions of the GPM parameters so that the contribution of the "quark sea" effects can be calculated explicitly. For the case of spin $(1 / 2)^{+}$octet baryons, we find that the quadrupole moments are zero for all the cases in NQM. Even if we consider the contribution coming from two-quark terms with the 
TABLE 10: Quadrupole moments of the spin $(3 / 2)^{+}$decuplet baryons in $\chi$ CQM using GPM and SU(3) symmetry breaking.

\begin{tabular}{|c|c|c|c|c|c|c|c|c|}
\hline \multirow{3}{*}{ Baryon } & \multirow{3}{*}{$\mathrm{NQM} \mathrm{fm}{ }^{2}$} & \multirow{3}{*}{$\begin{array}{c}\text { CQM [84] } \\
10^{-2} \mathrm{fm}^{2}\end{array}$} & \multirow{3}{*}{$\begin{array}{c}\chi \mathrm{PT}[99-103] \\
10^{-1} \mathrm{fm}^{2}\end{array}$} & \multirow{3}{*}{$\begin{array}{c}\text { SRA } \\
{[89,90]} \\
10^{-1} \mathrm{fm}^{2}\end{array}$} & \multirow{3}{*}{$\begin{array}{c}\text { Skyrme } \\
{[91,92]} \\
10^{-2} \mathrm{fm}^{2}\end{array}$} & \multirow{3}{*}{$\begin{array}{c}\text { GPM } \\
{[93,94]} \\
\mathrm{fm}^{2}\end{array}$} & \multicolumn{2}{|c|}{$\chi \mathrm{CQM}$ with SU(3) } \\
\hline & & & & & & & Symmetry & Symmetry breaking \\
\hline & & & & & & & $\mathrm{fm}^{2}$ & $\mathrm{fm}^{2}$ \\
\hline$\Delta^{++}$ & -0.409 & -9.3 & $-0.8 \pm 0.5$ & -0.87 & -8.8 & -0.12 & -0.3437 & -0.3695 \\
\hline$\Delta^{+}$ & -0.204 & -4.6 & $-0.3 \pm 0.2$ & -0.31 & -2.9 & -0.06 & -0.1719 & -0.1820 \\
\hline$\Delta^{0}$ & 0.0 & 0.0 & $0.12 \pm 0.05$ & 0.24 & 2.9 & 0.0 & 0.0 & 0.0055 \\
\hline$\Delta^{-}$ & 0.204 & 4.6 & $0.6 \pm 0.3$ & 0.80 & 8.8 & 0.06 & 0.1719 & 0.1930 \\
\hline$\Sigma^{*+}$ & -0.204 & -5.4 & $-0.7 \pm 0.3$ & -0.42 & -7.1 & -0.069 & -0.1719 & -0.1808 \\
\hline$\Sigma^{*-}$ & 0.204 & 4.0 & $4.0 \pm 0.2$ & 0.52 & 7.1 & 0.039 & 0.1719 & 0.1942 \\
\hline$\Sigma^{* 0}$ & 0.0 & -0.7 & $-0.13 \pm 0.07$ & 0.05 & 0.0 & 0.014 & 0.0 & 0.0067 \\
\hline$\Xi^{* 0}$ & 0.0 & -1.3 & $-0.35 \pm 0.2$ & -0.07 & -4.6 & -0.1719 & 0.0 & 0.0079 \\
\hline$\Xi^{*-}$ & 0.204 & 3.4 & $0.2 \pm 0.1$ & 0.35 & 4.6 & 0.024 & 0.1719 & 0.1954 \\
\hline$\Omega^{-}$ & 0.204 & 2.8 & $0.09 \pm 0.05$ & 0.24 & 0.0 & 0.014 & 0.1719 & 0.1966 \\
\hline
\end{tabular}

inclusion of "quark sea" and SU(3) symmetry breaking effects, the quadrupole moments still remain zero. In the case of $(3 / 2)^{+}$decuplet baryons, the quadrupole moments of the charged baryons are equal whereas all neutral baryons have zero quadrupole moment.

For the $(3 / 2)^{+}$decuplet and radiative decays of baryons, it can be easily shown that in the SU(3) symmetric limit, the magnitude of quadrupole moments can be expressed by the following relations:

$$
\begin{aligned}
& \frac{Q^{\Delta^{++}}}{2}=Q^{\Delta^{+}}=Q^{\Delta^{-}}=Q^{\Sigma^{*+}}=Q^{\Sigma^{*-}}=Q^{\Xi^{*-}}=Q^{\Omega^{-}}, \\
& Q^{\Delta^{+} p}=Q^{\Sigma^{*+} \Sigma^{+}}=2 Q^{\Sigma^{* 0} \Sigma^{0}}=Q^{\Xi^{* 0} \Xi^{0}}=\frac{2}{\sqrt{3}} Q^{\Sigma^{* 0} \Lambda} .
\end{aligned}
$$

The inclusion of SU(3) symmetry breaking changes this pattern considerably, and we obtain

$$
\begin{gathered}
Q^{\Omega^{-}}>Q^{\Xi^{*-}}>Q^{\Sigma^{*-}}>Q^{\Delta^{-}}>\frac{Q^{\Delta^{++}}}{2}>Q^{\Delta^{+}}>Q^{\Sigma^{*+}}, \\
2 Q^{\Sigma^{* 0} \Sigma^{0}}>Q^{\Xi^{* 0} \Xi^{0}}=Q^{\Sigma^{*+} \Sigma^{+}}>Q^{\Delta^{+}} p=\frac{2}{\sqrt{3}} Q^{\Sigma^{* 0} \Lambda} .
\end{gathered}
$$

Also we have

$$
Q^{\Xi^{* 0}}=Q^{\Sigma^{* 0}}=Q^{\Delta^{0}}
$$

which has its importance in the isospin limit where the three-quark core in neutral baryons does not contribute to the quadrupole moment. In the limit of SU(3) symmetry breaking, a nonvanishing value for the neutral baryons quadrupole moment is generated by the "quark sea" through the chiral fluctuations of constituent quarks leading to

$$
Q^{\Xi^{* 0}}>Q^{\Sigma^{* 0}}>Q^{\Delta^{0}}
$$

In the SU(3) limit, the transition moments involving the negatively charged baryons are zero:

$$
Q^{\Xi^{*-} \Xi^{-}}=Q^{\Sigma^{*-} \Sigma^{-}}=0
$$

This is because if flavor symmetry is exact, U-spin conservation forbids such transitions. The exact order of SU(3) symmetry breaking effects can be easily found from Tables 10 and 11. Since there is no experimental or phenomenological information available for any of these quadrupole moments, the accuracy of these relations can be tested by the future experiments.

For the spin $(3 / 2)^{+}$decuplet baryons presented in Table 10, quadrupole moments results in NQM using the GPM predict an oblate shape for all positively charged baryons $\left(\Delta^{++}, \Delta^{+}\right.$, and $\left.\Sigma^{*+}\right)$, prolate shape for negatively charged baryons $\left(\Delta^{-}, \Sigma^{*-}, \Xi^{*-}\right.$, and $\left.\Omega^{-}\right)$. It is important to mention here that the NQM is unable to explain the deformation in neutral baryons $\left(\Delta^{0}, \Sigma^{* 0}\right.$, and $\left.\Xi^{* 0}\right)$. On incorporating the effects of chiral symmetry breaking and "quark sea" in the $\chi \mathrm{CQM}$, a small amount of prolate deformation in neutral baryons $\left(\Delta^{0}, \Sigma^{* 0}\right.$, and $\left.\Xi^{* 0}\right)$ is observed. The trend of deformations is however the same for the positively and negatively charged baryons in $\chi \mathrm{CQM}$ and NQM. The other phenomenological models also observe a similar trend, for example, light cone QCD sum rules $[95,96]$, spectator quark model [86-88], Lattice QCD [104-108], $\chi$ PT [99-103], chiral quark soliton model ( $\chi \mathrm{QSM})[85]$, and so forth.

For the case of spin $(3 / 2)^{+} \rightarrow(1 / 2)^{+}$transitions in Table 11, it is observed that quadrupole moments of all the transitions are oblate in shape. This result is further endorsed by the predictions of Skyrme model $[91,92]$. The effects of chiral symmetry breaking can further be substantiated by a measurement of the other transition quadrupole moments.

\section{Summary and Conclusion}

To summarize, $\chi \mathrm{CQM}$ is able to provide a fairly good description of the charge radii of spin $(1 / 2)^{+}$octet and spin $(3 / 2)^{+}$decuplet baryons and quadrupole moments of $\operatorname{spin}(3 / 2)^{+}$decuplet baryons and spin $(3 / 2)^{+} \rightarrow(1 / 2)^{+}$ transitions using general parameterization method (GPM). The most significant prediction of the model for the charge 
TABLE 11: Quadrupole moments of the spin $(3 / 2)^{+} \rightarrow(1 / 2)^{+}$decuplet to octet transitions in $\chi$ CQM using GPM and SU(3) symmetry breaking.

\begin{tabular}{|c|c|c|c|c|c|}
\hline \multirow{2}{*}{ Baryon } & \multirow{2}{*}{$\mathrm{NQM} \mathrm{fm}^{2}$} & \multirow{2}{*}{ Skyrme $[91,92] 10^{-2} \mathrm{fm}^{2}$} & \multirow{2}{*}{ GPM $[93,94] \mathrm{fm}^{2}$} & \multicolumn{2}{|c|}{$\chi \mathrm{CQM}$ with SU(3) } \\
\hline & & & & Symmetry fm ${ }^{2}$ & Symmerty breaking $\mathrm{fm}^{2}$ \\
\hline$\Delta^{+} p$ & -0.110 & -5.2 & -0.082 & -0.0608 & -0.0846 \\
\hline \multicolumn{6}{|c|}{$-0.0846 \pm 0.0033$} \\
\hline$\Sigma^{*+} \Sigma^{+}$ & -0.110 & -0.93 & -0.076 & -0.0608 & -0.0864 \\
\hline$\Sigma^{*-} \Sigma^{-}$ & 0.0 & 0.93 & 0.014 & -0.0608 & -0.0018 \\
\hline$\Sigma^{* 0} \Sigma^{0}$ & -0.055 & 0.0 & -0.031 & 0.0 & -0.0441 \\
\hline$\Xi^{* 0} \Xi^{0}$ & -0.110 & 2.91 & -0.031 & -0.0608 & -0.0864 \\
\hline$\Xi^{*-} \Xi^{-}$ & 0.0 & -2.91 & 0.007 & 0.0 & -0.0018 \\
\hline$\Sigma^{* 0} \Lambda$ & -0.096 & -4.83 & -0.041 & -0.0526 & -0.0733 \\
\hline
\end{tabular}

radii is the nonzero value pertaining to the neutral octet baryons $\left(n, \Sigma^{0}, \Xi^{0}\right.$, and $\left.\Lambda\right)$ and decuplet baryons $\left(\Delta^{0}, \Sigma^{* 0}\right.$, $\left.\Xi^{* 0}\right)$. For the quadrupole moment, prolate shape is observed for the spin $(3 / 2)^{+}$neutral decuplet baryons $\left(\Delta^{0}, \Sigma^{* 0}\right.$, and $\left.\Xi^{* 0}\right)$. The effects of SU(3) symmetry breaking have also been investigated, and the results show considerable improvement over the SU(3) symmetric case. We have also studied the implications of GPM parameters, particularly, the contribution of the three-quark term in the octet and decuplet baryon. We find that the sign of the three-quark term contribution is opposite in the case of octet and decuplet baryons charge radii. New experiments aimed at measuring the charge radii and quadrupole moment of the other baryons are needed for a profound understanding of the hadron structure in the nonperturbative regime of QCD.

In conclusion, we would like to state that at-the-leadingorder constituent quarks and the weakly interacting Goldstone bosons constitute the appropriate degrees of freedom in the nonperturbative regime of QCD. The SU(3) symmetry breaking parameters pertaining to the strangeness contribution and the GPM parameters pertaining to the one-, two-, and three-quark contributions are the key in understanding the octet and decuplet baryon charge radii and quadrupole moment.

\section{Acknowledgments}

The authors would like to thank the Department of Atomic Energy, Government of India for the financial support through Grant no. 2010/37P/48/BRNS/1445.

\section{References}

[1] R. G. Sachs, "High-energy behavior of nucleon electromagnetic form factors," Physical Review, vol. 126, no. 6, pp. 2256-2260, 1962.

[2] J. Ashman, B. Badelek, G. Baum et al., "A measurement ofthe spin asymmetry and determination of the structure function $g_{1}$ in deep inelastic muon-proton scattering," Physics Letters B, vol. 206, no. 2, pp. 364-370, 1988.

[3] J. Ashman, B. Badelek, G. Baum et al., "An investigation of the spin structure of the proton in deep inelastic scattering of polarised muons on polarised protons," Nuclear Physics B, vol. 328, no. 1, pp. 1-35, 1989.

[4] J. J. Kelly, "Nucleon charge and magnetization densities from Sachs form factors," Physical Review C, vol. 66, no. 6, Article ID 065203, 2002.

[5] J. Friedrich and T. Walcher, "A coherent interpretation of the form factors of the nucleon in terms of a pion cloud and constituent quarks," The European Physical Journal A, vol. 17, pp. 607-623, 2003.

[6] S. L. Glashow, “The unmellisonant quark," Physica A, vol. 96, no. 1-2, pp. 27-30, 1979.

[7] S. Taylor, G. S. Mutchler, G. Adams et al., "Radiative decays of the $\Sigma^{0}(1385)$ and $\Lambda(1520)$ hyperons," Physical Review C, vol. 71, no. 5, Article ID 054609, 2005.

[8] O. Gayou, K. Wijesooriya, A. Afanasev et al., "Measurements of the elastic electromagnetic form factor ratio $\mu_{p} G_{E p} / G_{M p}$ via polarization transfer," Physical Review $C$, vol. 64, no. 3, Article ID 038202, 2001.

[9] O. Gayou, K. A. Aniol, T. Averett et al., "Measurement of $G_{E p} / G_{M p}$ in $e^{\rightarrow} p \rightarrow e p \rightarrow$ to $Q^{2}=5.6 \mathrm{GeV}^{2}$, "Physical Review Letters, vol. 88, no. 9, Article ID 092301, 2002.

[10] V. Punjabi, C. F. Perdrisat, K. A. Aniol et al., "Proton elastic form factor ratios to $\mathrm{Q}^{2}=3.5 \mathrm{GeV}^{2}$ by polarization transfer," Physical Review C, vol. 71, Article ID 055202, 2005.

[11] C. B. Crawford, A. Sindile, T. Akdogan et al., "Measurement of the proton's electric to magnetic form factor ratio from ${ }^{1} H^{\rightarrow}\left(e^{\rightarrow}, e^{\prime} p\right), "$ Physical Review Letters, vol. 98, no. 5, Article ID 052301, 2007.

[12] K. Nakamura, "Review of particle physics," Journal of Physics G, vol. 37, no. 7, Article ID 075021, 2010.

[13] I. Eschrich, H. Krügeri, J. Simon et al., "Measurement of the $S^{-}$ charge radius by $S^{-}$-electron elastic scattering," Physics Letters $B$, vol. 522, no. 3-4, pp. 233-239, 2001.

[14] M. I. Adamovich, Y. A. Alexandrov, S. P. Baranov et al., "First observation of $S^{-}-e^{-}$elastic scattering in the hyperon beam experiment WA89 at CERN," The European Physical Journal C, vol. 8, no. 1, pp. 59-66, 1999.

[15] R. Rosenfelder, "Coulomb corrections to elastic electron-proton scattering and the proton charge radius," Physics Letters B, vol. 479, no. 4, pp. 381-386, 2000.

[16] G. Blanpied, M. Blecher, A. Caracappa et al., " $N \rightarrow \Delta$ Transition from simultaneous measurements of $p\left(\gamma^{\rightarrow}, \pi\right)$ and $p\left(\gamma^{\rightarrow}, \gamma\right)$, , Physical Review Letters, vol. 79, pp. 4337-4340, 1997. 
[17] R. Beck, H. P. Krahn, J. Ahrens et al., "Measurement of the $E 2 / M 1$ Ratio in the $N \rightarrow \Delta$ Transition using the reaction $p\left(\gamma^{\rightarrow}, p\right) \pi^{o}$, Physical Review Letters, vol. 78, no. 4, pp. 606-609, 1997.

[18] G. Blanpied, M. Blecher, A. Caracappa et al., " $N \rightarrow \Delta$ transition and proton polarizabilities from measurements of $p\left(\gamma^{\rightarrow}, \gamma\right), p\left(\gamma^{\rightarrow}, \pi^{o}\right)$ and $p\left(\gamma^{\rightarrow}, \pi^{+}\right), "$ Physical Review $C$, vol. 64, no. 2, Article ID 025203, 2001.

[19] L. Tiator, D. Drechsel, S. S. Kamalov, and S. N. Yang, "Electromagnetic form factors of the $\Delta$ (1232) excitation," The European Physical Journal A, vol. 17, no. 3, pp. 357-363, 2003.

[20] C. Becchi and G. Morpurgo, "Vanishing of the E2 part of the $\mathrm{N}^{*} 33 \rightarrow \mathrm{N}+\gamma$ amplitude in the non-relativistic quark model of "elementary" particles," Physics Letters, vol. 17, no. 3, pp. 352354, 1965.

[21] A. M. Bernstein, "Deviation of the nucleon shape from spherical symmetry: experimental status," The European Physical Journal A, vol. 17, no. 3, pp. 349-355, 2003.

[22] C. N. Papanicolas, "Nucleon deformation: a status report," The European Physical Journal A, vol. 18, no. 2-3, pp. 141-145, 2003.

[23] M. Gell-Mann, "A schematic model of baryons and mesons," Physics Letters, vol. 8, no. 3, pp. 214-215, 1964.

[24] R. P. Feynman, M. Gell-Mann, and G. Zweig, "Group U(6)âU(6) generated by current components," Physical Review Letters, vol. 13, no. 22, pp. 678-680, 1964.

[25] G. Zweig, "An model for strong interaction symmetry and its breaking I," CERN Reports 8182/TH401, 1964.

[26] G. Zweig, "An model for strong interaction symmetry and its breaking II," CERN Reports 8419/TH412, 1964.

[27] G. Morpurgo, "Is a nonrelativistic approximation possible for the internal dynamics of 'elementary' particles?" Physics, vol. 2, pp. 95-104, 1965.

[28] A. De Rújula, H. Georgi, and S. L. Glashow, "Hadron masses in a gauge theory," Physical Review D, vol. 12, no. 1, pp. 147-162, 1975.

[29] N. Isgur and G. Karl, "Ground-state baryon magnetic moments," Physical Review D, vol. 21, no. 11, pp. 3175-3179, 1980.

[30] N. Isgur, G. Karl, and D. W. L. Sprung, "Neutron charge form factor in a quark model with hyperfine interactions," Physical Review D, vol. 23, pp. 163-164, 1981.

[31] N. Isgur, G. Karl, and R. Koniuk, "D waves in the nucleon: a test of color magnetism," Physical Review D, vol. 25, no. 9, pp. 2394-2398, 1982.

[32] P. Geiger and N. Isgur, "Strange hadronic loops of the proton: a quark model calculation," Physical Review D, vol. 55, no. 1, pp. 299-310, 1997.

[33] A. L. Yaouanc, L. Oliver, O. Pène, and J. C. Raynal, "Combined effects of internal quark motion and $\mathrm{SU}(6)$ breaking on the properties of the baryon ground state," Physical Review D, vol. 15 , no. 3, pp. 844-853, 1977.

[34] M. Gupta and A. N. Mitra, "Even-wave harmonic-oscillator theory of baryonic states. IV. Structure functions versus slope of neutron charge form factor," Physical Review D, vol. 18, no. 5, pp. 1585-1590, 1978.

[35] M. Gupta, S. K. Sood, and A. N. Mitra, "Resonance photocouplings with a "mixed" nucleon and the even-wave harmonicoscillator theory of baryonic states," Physical Review D, vol. 16, no. 1, pp. 216-219, 1977.

[36] M. Gupta, S. K. Sood, and A. N. Mitra, "Even-wave harmonicoscillator theory of baryonic states. V. Polarized photoproduction of single pions," Physical Review D, vol. 19, pp. 104-111, 1979.
[37] P. Amaudruz, M. Arneodo, A. Arvidson et al., "Gottfried sum from the ratio $F_{2}^{n} / F_{2}^{p}$," Physical Review Letters, vol. 66, no. 21, pp. 2712-2715, 1991.

[38] M. Arneodo, A. Arvidson, B. Badelek et al., "Reevaluation of the Gottfried sum," Physical Review D, vol. 50, no. 1, pp. R1-R3, 1994.

[39] E. A. Hawker, T. C. Awes, M. E. Beddo et al., "Measurement of the light antiquark flavor asymmetry in the nucleon sea," Physical Review Letters, vol. 80, no. 17, pp. 3715-3718, 1998.

[40] J. C. Peng, G. T. Garvey, T. C. Awes et al., " $\bar{d} / \bar{u}$ asymmetry and the origin of the nucleon sea," Physical Review D, vol. 58, no. 9, Article ID 092004, 1998.

[41] R. S. Towell, P. L. McGaughey, T. C. Awes et al., "Improved measurement of the $\bar{d} / \bar{u}$ asymmetry in the nucleon sea," Physical Review D, vol. 64, no. 5, Article ID 052002, 2001.

[42] J. Kunz and P. J. Mulders, "Electromagnetic properties of hypersons in the bound-state approach to the Skyrme model," Physical Review D, vol. 41, no. 5, pp. 1578-1585, 1990.

[43] J. Kunz, P. J. Mulders, and G. A. Miller, "Charge distributions of hyperons," Physics Letters B, vol. 255, no. 1, pp. 11-17, 1991.

[44] C. Gobbi, S. Boffi, and D. O. Riska, "Mean square radii of hyperons in the bound-state soliton model," Nuclear Physics A, vol. 547, no. 4, pp. 633-644, 1992.

[45] B. Schwesinger and H. Weigel, "SU(3) symmetry breaking for masses, magnetic moments and sizes of baryons," Nuclear Physics A, vol. 540, no. 3-4, pp. 461-477, 1992.

[46] H. Kim, A. Blotz, M. V. Polyakov, and K. Goeke, "Electromagnetic form factors of the $\mathrm{SU}(3)$ octet baryons in the semibosonized SU(3) Nambu-Jona-Lasinio model," Physical Review D, vol. 53, no. 7, pp. 4013-4029, 1996.

[47] D. H. Lu, A. W. Thomas, and A. G. Williams, "Electromagnetic form factors of the nucleon in an improved quark model," Physical Review C, vol. 57, no. 5, pp. 2628-2637, 1998.

[48] G. Wagner, A. J. Buchmann, and A. Faessler, "Exchange currents in octet hyperon charge radii," Physical Review C, vol. 58, no. 6, pp. 3666-3669, 1998.

[49] N. Barik, S. N. Jena, and D. P. Rath, "Nucleon form factors and static properties of baryons in a quark model," Physical Review $D$, vol. 41, no. 5, pp. 1568-1577, 1990.

[50] M. Warns, W. Pfeil, and H. Rollnik, "Electromagnetic properties of hyperons in a relativised quark model," Physics Letters B, vol. 258, no. 3-4, pp. 431-440, 1991.

[51] T. V. Cauteren, D. Merten, T. Corthals et al., "Electric and magnetic form factors of strange baryons," The European Physical Journal A, vol. 20, no. 2, pp. 283-291, 2004.

[52] K. Berger, R. F. Wagenbrunn, and W. Plessas, "Covariant baryon charge radii and magnetic moments in a chiral constituentquark model," Physical Review D, vol. 70, no. 9, Article ID 094027, 2004.

[53] B. J. Diaz, D. O. Riska, and F. Coester, "Baryon form factors of relativisitic constituent-quark models," Physical Review C, vol. 69, Article ID 035212, 2004.

[54] B. J. Diaz, D. O. Riska, and F. Coester, "Erratum: baryon form factors of relativisitic constituent-quark models," Physical Review C, vol. 75, Article ID 069902, 2007.

[55] A. J. Buchmann and R. F. Lebed, "Large $N_{c}$, constituent quarks, and $N, \Delta$ charge radii," Physical Review D, vol. 62, no. 9, Article ID 096005, 2000.

[56] A. J. Buchmann, "Charge form factors and nucleon shape," AIP Conference Proceedings, vol. 904, pp. 110-125. 
[57] A. J. Buchmann and R. F. Lebed, "Baryon charge radii and quadrupole moments in the $1 / N_{c}$ expansion: the 3-flavor case," Physical Review D, vol. 67, Article ID 016002, 17 pages, 2003.

[58] A. J. Buchmann, "Structure of strange baryons," in Proceedings of the International Conference on Hypernuclear and Strange Particle Physics (HYP '06), Mainz, Germany, October 2006.

[59] S. Cheedket, V. E. Lyubovitskij, T. Gutsche, A. Faessler, K. Pumsa-Ard, and Y. Yan, "Electromagnetic form factors of the baryon octet in the perturbative chiral quark model," The European Physical Journal A, vol. 20, no. 2, pp. 317-327, 2004.

[60] S. J. Puglia, M. J. R. Musolf, and S. L. Zhu, "Octet baryon charge radii, chiral symmetry, and decuplet intermediate states," Physical Review D, vol. 36, Article ID 034014, 2001.

[61] B. Kubis and U. G. Meissner, "Baryon form factors in chiral perturbation theory," The European Physical Journal C, vol. 18, no. 4, pp. 747-756, 2001.

[62] D. Arndt and B. C. Tiburzi, "Charge radii of the meson and baryon octets in quenched and partially quenched chiral perturbation theory," Physical Review D, vol. 68, no. 9, Article ID 094501, 2003.

[63] P. Wang, D. B. Leinweber, A. W. Thomas, and R. D. Young, "Chiral extrapolation of octet-baryon charge radii," Physical Review D, vol. 79, no. 9, Article ID 094001, 2009.

[64] D. B. Leinweber, T. Draper, and R. M. Woloshyn, "Decuplet baryon structure from lattice QCD," Physical Review D, vol. 46, no. 7, pp. 3067-3085, 1992.

[65] D. Arndt and B. C. Tiburzi, "Electromagnetic properties of the baryon decuplet in quenched and partially quenched chiral perturbation theory," Physical Review D, vol. 68, Article ID 114503, 2003.

[66] S. Boinepalli, D. B. Leinweber, P. J. Moran, A. G. Williams, J. M. Zanotti, and J. B. Zhang, "Electromagnetic structure of decuplet baryons towards the chiral regime," Physical Review D, vol. 80, no. 5, Article ID 054505, 2009.

[67] R. K. Sahoo, A. R. Panda, and A. Nath, "Magnetic moments and charge radii of decuplet baryons in a field-theoretic quark model," Physical Review D, vol. 52, no. 7, pp. 4099-4105, 1995.

[68] L. S. Geng, J. M. Camalich, and M. J. V. Vacas, "Electromagnetic structure of the lowest-lying decuplet resonances in covariant chiral perturbation theory," Physical Review D, vol. 80, no. 3, Article ID 034027, 2009.

[69] C. Alexandrou, P. de Forcrand, T. Lippert et al., " $N$ to $\Delta$ electromagnetic transition form factors from lattice QCD," Physical Review D, vol. 69, no. 11, Article ID 114506, 2004.

[70] C. Alexandrou, P. de Forcrand, H. Neff et al., " $N$ to $\Delta$ electromagnetic-transition form factors from lattice QCD," Physical Review Letters, vol. 94, no. 2, Article ID 021601, 2005.

[71] G. Clement and M. Maamache, "Are the $N$ and $\Delta$ deformed MIT bags?” Annals of Physics, vol. 165, no. 1, pp. 1-16, 1985.

[72] A. B. Migdal, “Traektorii Redzhe i forma adronov," Pis'Ma V ZhETF, vol. 46, no. 7, pp. 256-258, 1987.

[73] A. B. Migdal, "Regge trajectories and the shape of hadrons," JETP Letters, vol. 46, no. 7, pp. 322-325, 1987.

[74] A. J. Buchmann, " $N$-to- $\Delta$ quadrupole transition in the constituent quark model," in Proceedings of the 8th International Conference on the Structure of Baryons (Baryons' 98), D. W. Menze and B. Metsch, Eds., p. 731, World Scientific, Singapore, 1999.

[75] A. J. Buchmann, E. Hernandez, and A. Faessler, "Electromagnetic properties of the $\Delta$ (1232)," Physical Review C, vol. 55, no. 1, pp. 448-463, 1997.
[76] A. J. Buchmann and E. M. Henley, "Intrinsic quadrupole moment of the nucleon," Physical Review D, vol. 63, Article ID 015202, 2001.

[77] A. J. Buchmann and E. M. Henley, "Quadrupole moments of baryons," Physical Review D, vol. 65, no. 7, Article ID 073017, 2002.

[78] A. J. Buchmann, J. A. Hester, and R. F. Lebed, "Quadrupole moments of $\mathrm{N}$ and $\Delta$ in the $1 / N_{c}$ expansion," Physical Review $D$, vol. 66, no. 5, Article ID 056002, 2002.

[79] A. J. Buchmann, "Size and shape of baryons in a large $N_{c}$ quark model," in Proceedings of the Institute for Nuclear Theory, Phenomenology of Large NcQCD, R. F. Lebed, Ed., vol. 12, p. 224, World Scientific, 2002.

[80] A. J. Buchmann and R. F. Lebed, "Baryon charge radii and quadrupole moments in the $1 / N_{c}$ expansion: the 3-flavor case," Physical Review D, vol. 67, Article ID 016002, 2003.

[81] N. Isgur, G. Karl, and R. Koniuk, "Dwaves in the nucleon: a test of color magnetism," Physical Review D, vol. 25, pp. 2394-2398, 1982.

[82] W. J. Leonard and W. J. Gerace, "Quark-meson coupling model for baryon wave functions and properties," Physical Review D, vol. 41, pp. 924-936, 1990.

[83] G. Wagner, A. J. Buchmann, and A. Faessler, "Electromagnetic properties of decuplet hyperons in a chiral quark model with exchange currents," Journal of Physics G, vol. 26, no. 3, article $267,2000$.

[84] M. I. Krivoruchenko and M. M. Giannini, "Quadrupole moments of the decuplet baryons," Physical Review D, vol. 43, no. 11, pp. 3763-3765, 1991.

[85] T. Ledwig, A. Silva, and M. Vanderhaeghen, "Electromagnetic properties of the $\Delta(1232)$ and decuplet baryons in the selfconsistent SU(3) chiral quark-soliton model," Physical Review $D$, vol. 79, no. 9, Article ID 094025, 2009.

[86] G. Ramalho and M. T. Pena, "Electromagnetic form factors of the $\Delta$ in an S-wave approach," Journal of Physics G, vol. 36, no. 8, Article ID 085004, 2009.

[87] G. Ramalho, M. T. Pena, and F. Gross, "Electric quadrupole and magnetic octupole moments of the $\Delta$," Physics Letters B, vol. 678, no. 4, pp. 355-358, 2009.

[88] G. Ramalho, M. T. Pena, and F. Gross, "Electromagnetic form factors of the $\Delta$ with D-waves," Physical Review D, vol. 81, no. 11, Article ID 113011, 2010.

[89] B. Schwesinger and H. Weigel, "SU(3) symmetry breaking for masses, magnetic moments and sizes of baryons," Nuclear Physics A, vol. 540, no. 3-4, pp. 461-477, 1992.

[90] J. Kroll and B. Schwesinger, "Electric quadrupole moments of the decuplet and the strangeness content of the proton," Physics Letters B, vol. 334, pp. 287-289, 1994.

[91] Y. Oh, "Electric quadrupole moments of the decuplet baryons in the Skyrme model," Modern Physics Letters A, vol. 10, pp. 10271034, 1995.

[92] A. Abada, H. Weigel, and H. Reinhardt, "Radiative decays of hyperons in the Skyrme model: view the MathML source transitions ratios E2/M1," Physics Letters B, vol. 366, pp. 26-31, 1996.

[93] A. J. Buchmann and E. M. Henley, "Quadrupole moments of baryons," Physical Review D, vol. 65, no. 7, Article ID 073017, 2002.

[94] A. J. Buchmann and E. M. Henley, "Baryon octupole moments," The European Physical Journal A, vol. 35, no. 3, pp. 267-269, 2008. 
[95] K. Azizi, "Magnetic dipole, electric quadrupole and magnetic octupole moments of the $\Delta$ baryons in light cone QCD sum rules," The European Physical Journal C, vol. 61, no. 2, pp. 311319, 2009.

[96] T. M. Aliev, K. Azizi, and M. Savci, "Electric quadrupole and magnetic octupole moments of the light decuplet baryons within light cone QCD sum rules," Physics Letters, vol. 681, no. 3, pp. 240-246, 2009.

[97] A. J. Buchmann, J. A. Hester, and R. F. Lebed, "Quadrupole moments of $\mathrm{N}$ and $\Delta$ in the $1 / N_{c}$ expansion," Physical Review $D$, vol. 66, no. 5, Article ID 056002, 2002.

[98] A. J. Buchmann and R. F. Lebed, "Baryon charge radii and quadrupole moments in the $1 / N_{c}$ expansion: the 3-flavor case," Physical Review D, vol. 67, Article ID 016002, 2003.

[99] M. N. Butler, M. J. Savage, and R. P. Springer, "Electromagnetic moments of the baryon decuplet," Physical Review D, vol. 49, pp. 3459-3465, 1994.

[100] M. K. Banerjee and J. Milana, "Decuplet reexamined in chiral perturbation theory," Physical Review D, vol. 54, no. 9, pp. 58045811, 1996.

[101] D. Arndt and B. C. Tiburzi, "Electromagnetic properties of the baryon decuplet in quenched and partially quenched chiral perturbation theory," Physical Review D, vol. 68, no. 11, Article ID 114503, 2003.

[102] D. Arndt and B. C. Tiburzi, "Erratum: electromagnetic properties of the baryon decuplet in quenched and partially quenched chiral perturbation theory," Physical Review D, vol. 65, no. 5, Article ID 059904, 2004.

[103] L. S. Geng, J. M. Camalich, and M. J. V. Vacas, "Electromagnetic structure of the lowest-lying decuplet resonances in covariant chiral perturbation theory," Physical Review D, vol. 80, Article ID 034027, 2009.

[104] D. B. Leinweber, T. Draper, and R. M. Woloshyn, "Decuplet baryon structure from lattice QCD," Physical Review D, vol. 46, pp. 3067-3085, 1992.

[105] C. Alexandrou, T. Korzec, T. Leontiou, J. W. Negele, and A. Tsapalis, "Electromagnetic form factors of the Delta baryon," PoSLATTICE, 2007, p. 149, 2006.

[106] C. Alexandrou, T. Korzec, G. Koutsou et al., "Quark transverse charge densities in the delta-(1232) from lattice QCD," Nuclear Physics A, vol. 825, no. 1-2, pp. 115-144, 2009.

[107] C. Alexandrou, T. Korzec, G. Koutsou et al., "Delta-baryon electromagnetic form factors in lattice QCD," Physical Review $D$, vol. 79, Article ID 014507, 2009.

[108] S. Boinepalli, D. B. Leinweber, P. J. Moran, A. G. Williams, J. M. Zanotti, and J. B. Zhang, "Electromagnetic structure of decuplet baryons towards the chiral regime," Physical Review D, vol. 80, Article ID 054505, 2009.

[109] S. Weinberg, "Phenomenological lagrangians," Physica A, vol. 96, no. 1-2, pp. 327-340, 1979.

[110] A. Manohar and H. Georgi, "Chiral quarks and the nonrelativistic quark model," Nuclear Physics B, vol. 234, no. 1, pp. 189-212, 1984.

[111] T. P. Cheng and L. F. Li, "Flavor and spin contents of the nucleon in the quark model with chiral symmetry," Physical Review Letters, vol. 74, pp. 2872-2875, 1995.

[112] T. P. Cheng and L. F. Li, "Chiral quark model of nucleon spinflavor structure with SU(3) and axial-U(1) breakings," Physical Review D, vol. 57, pp. 344-349, 1998.

[113] X. Song, J. S. McCarthy, and H. J. Weber, "Nucleon spin-flavor structure in the SU(3)-breaking chiral quark model," Physical Review D, vol. 55, no. 5, pp. 2624-2629, 1997.
[114] X. Song, "New relations and constraints on quark spin-flavor content in the symmetry-breaking chiral quark model," Physical Review D, vol. 57, pp. 4114-4123, 1998.

[115] J. Linde, T. Ohlsson, and H. Snellman, "Octet baryon magnetic moments in the chiral quark model with configuration mixing," Physical Review D, vol. 57, pp. 452-464, 1998.

[116] J. Linde, T. Ohlsson, and H. Snellman, "Decuplet baryon magnetic moments in the chiral quark model," Physical Review D, vol. 57, no. 9, pp. 5916-5919, 1998.

[117] H. Dahiya and M. Gupta, "Chiral quark model with configuration mixing," Physical Review D, vol. 64, Article ID 014013, 2001.

[118] H. Dahiya and M. Gupta, "Chiral quark model ( $\chi \mathrm{QM})$ and the nucleon spin," International Journal of Modern Physics A, vol. 19, no. 29, Article ID 5027, 2004.

[119] H. Dahiya and M. Gupta, "Chiral constituent quark model and the coupling strength of $\eta$," International Journal of Modern Physics A, vol. 21, no. 21, Article ID 4255, 2006.

[120] N. Sharma, H. Dahiya, P. K. Chatley, and M. Gupta, "Weak vector and axial-vector form factors in the chiral constituent quark model with configuration mixing," Physical Review D, vol. 79, Article ID 077503, 2009.

[121] N. Sharma, H. Dahiya, and P. K. Chatley, "Extraction of the CKM matrix element Vus from the hyperon semileptonic decays," The European Physical Journal A, vol. 44, no. 1, pp. 125$128,2010$.

[122] H. Dahiya and M. Gupta, " $x$-dependence of the quark distribution functions in the $\chi \mathrm{CQM}$," The European Physical Journal C, vol. 52, no. 3, pp. 571-579, 2007.

[123] H. Dahiya and M. Gupta, "Spin and flavor strange quark content of the nucleon," Physical Review D, vol. 78, Article ID 014001, 2008.

[124] N. Sharma and H. Dahiya, "Quark sea asymmetries of the octet baryons," Physical Review D, vol. 81, Article ID 114003, 2010.

[125] H. Dahiya and M. Gupta, "Octet magnetic moments and the Coleman-Glashow sum rule violation in the chiral quark model," Physical Review D, vol. 66, Article ID 051501, 2002.

[126] H. Dahiya and M. Gupta, "Octet and decuplet baryon magnetic moments in the chiral quark model," Physical Review D, vol. 67, p. 114015, 2003.

[127] H. Dahiya and M. Gupta, "SU(4) chiral quark model with configuration mixing," Physical Review D, vol. 67, Article ID 074001, 2003.

[128] N. Sharma, A. M. Torres, K. P. Khemchandani, and H. Dahiya, "Magnetic moments of the low-lying $(1 / 2)^{-}$octet baryon resonances," The European Physical Journal A, vol. 49, article 11, 2013.

[129] A. M. Torres, K. P. Khemchandani, N. Sharma, and H. Dahiya, "Magnetic moments of the low-lying JP $=(1 / 2)^{-},(3 / 2)^{-} \wedge$ resonances within the framework of the chiral quark model," The European Physical Journal A, vol. 48, article 185, 2012.

[130] N. Sharma and H. Dahiya, "Charge radii of octet and decuplet baryons," in AIP Conference Proceedings, vol. 1388, pp. 458-460, 2011.

[131] N. Sharma and H. Dahiya, "Quadrupole moments of low-lying baryons with spin- $(1 / 2)^{+}$, spin- $(3 / 2)^{+}$, and spin- $(3 / 2)^{+}$to $(1 / 2)^{+}$ transitions," Pramana, vol. 80, no. 2, pp. 237-249, 2013.

[132] H. Dahiya and M. Gupta, "SU(4) chiral quark model with configuration mixing," Physical Review D, vol. 67, no. 7, Article ID 074001, 2003.

[133] N. Sharma, H. Dahiya, P. K. Chatley, and M. Gupta, "Spin $(1 / 2)^{+}$, spin $(3 / 2)^{+}$, and transition magnetic moments of low lying and 
charmed baryons," Physical Review D, vol. 81, no. 7, Article ID 073001, 2010.

[134] G. Morpurgo, "Field theory and the nonrelativistic quark model: a parametrization of the baryon magnetic moments and masses," Physical Review D, vol. 40, pp. 2997-3011, 1989.

[135] G. Morpurgo, "Field theory and the nonrelativistic quark model: a parametrization of the meson masses," Physical Review D, vol. 41, no. 9, pp. 2865-2870, 1990.

[136] G. Dillon and G. Morpurgo, "Relation of constituent quark models to QCD: why several simple models work 'so well,"' Physical Review D, vol. 53, pp. 3754-3769, 1996.

[137] G. Dillon and G. Morpurgo, "Chiral QCD, general QCD parametrization, and constituent quark models," Physical Review D, vol. 68, Article ID 014001, 2003.

[138] G. Dillon and G. Morpurgo, "Baryon octet magnetic moments to all orders in flavor breaking: an application to the problem of the strangeness in the nucleon," Physical Review D, vol. 75, no. 7, Article ID 073007, 2007.

[139] A. J. Buchmann and E. M. Henley, "Intrinsic quadrupole moment of the nucleon," Physical Review C, vol. 63, no. 1, Article ID 015202, 8 pages, 2000.

[140] A. J. Buchmann, "Electromagnetic $N \rightarrow \Delta$ transition and neutron form factors," Physical Review Letters, vol. 93, Article ID 212301, 4 pages, 2004.

[141] A. J. Buchmann, "Structure of strange baryons," in Proceedings of the Shape of Hadrons Workshop, C. N. Papanicolas and A. M. Bernstein, Eds., Athens, Greece, 2006.

[142] A. J. Buchmann, "Charge form factors and nucleon shape," AIP Conference Proceedings, vol. 904, pp. 110-125, 2007.

[143] G. Morpurgo, "Smallness of gluon coupling to constituent quarks in baryons and validity of nonrelativistic quark model," Physical Review D, vol. 46, no. 9, pp. 4068-4075, 1992.

[144] G. Dillon and G. Morpurgo, "General QCD parametrization of the charge radii of $p, n$ and $\Delta^{+}$," Physics Letters $B$, vol. 448, no. 1-2, pp. 107-110, 1999. 

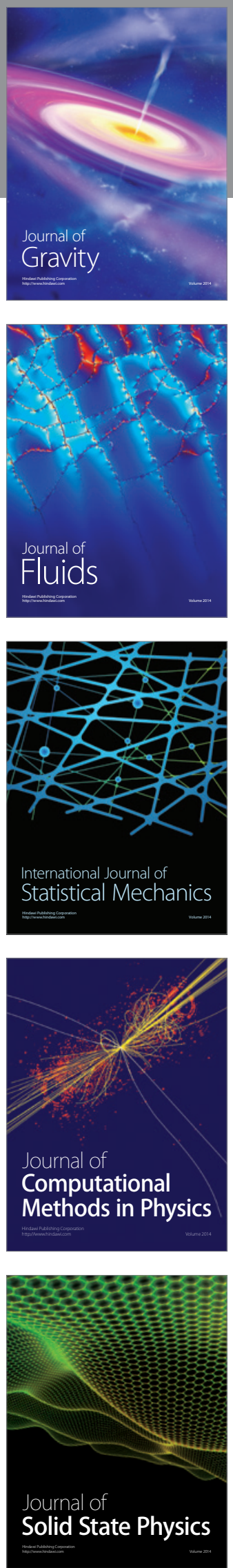

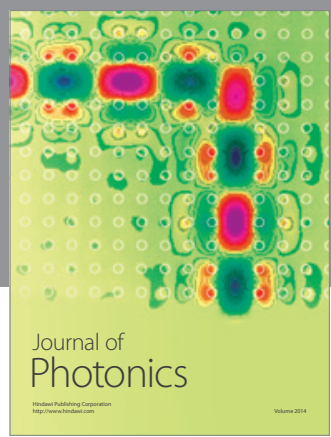

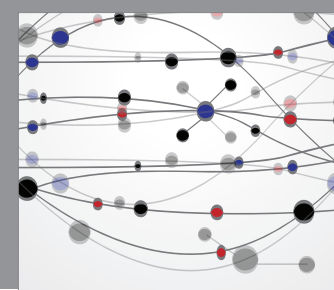

The Scientific World Journal

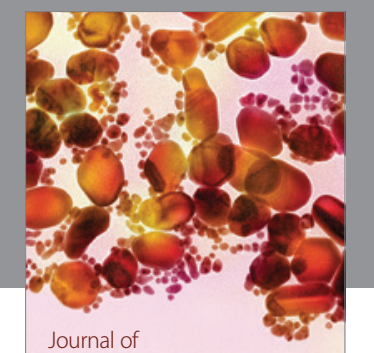

Soft Matter
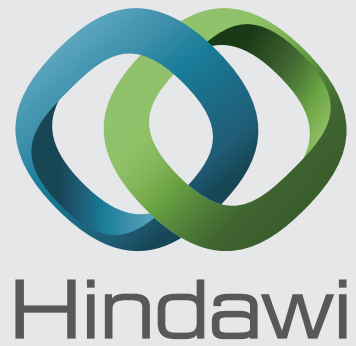

Submit your manuscripts at

http://www.hindawi.com
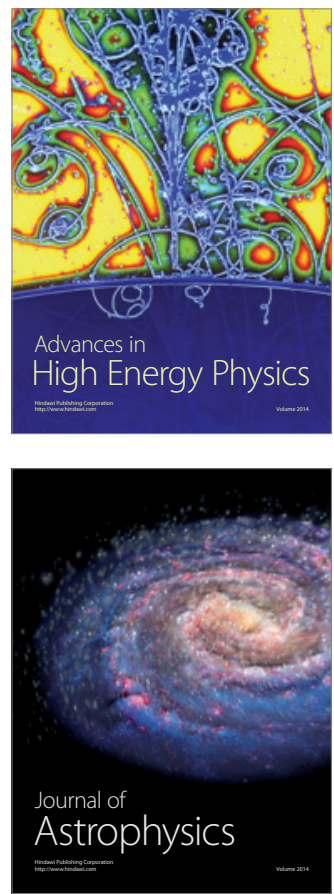
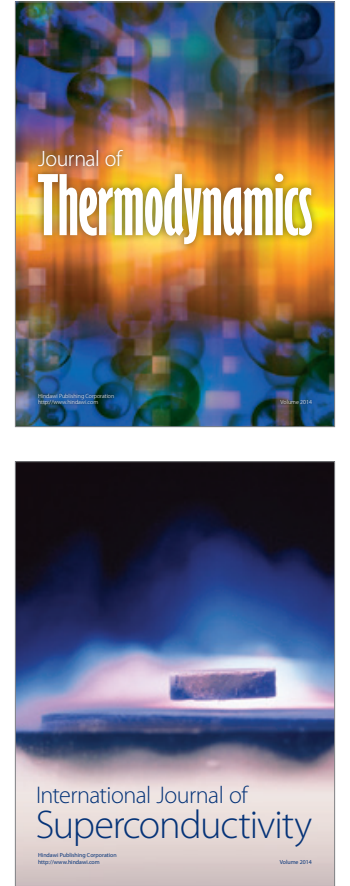
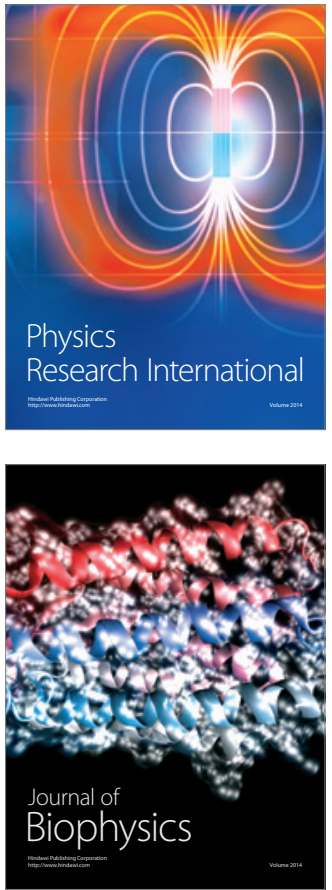
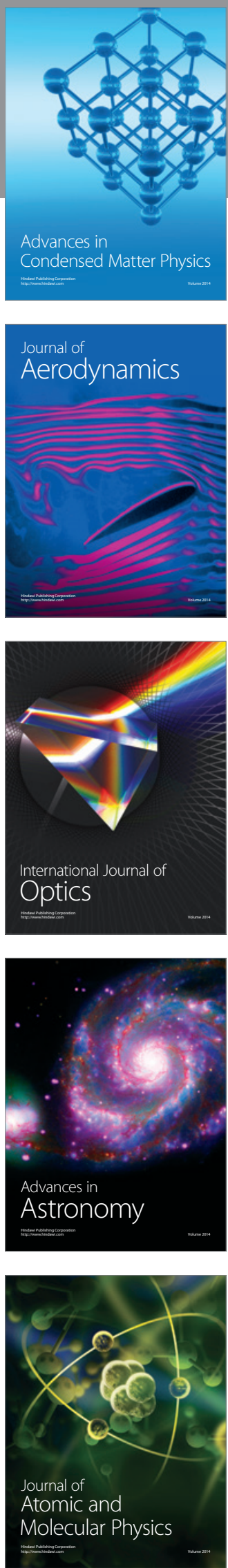